\title{
UNIVERSTCY OF VIRGINIA PUBLICATIONS
}

\section{BULLETIN}

OF THE

\section{PHILOSOPHICAL SOCIETY}

Saientifio Sories, Vox. I. No. 4, pp. $85-120 ;$ Jano, 1910

\section{NEW POSITIONS OF THE STARS IN THE HUYGHENIAN REGION OF THE GRTA NEBULA IN ORION}

From Observatione Made at the Loander MeCormick Obervatory

BT

RALPII E. WLSON 


\section{UNIVERSITY OF VIRGINIA. PUBLICATIONS}

The University of Virginia Bulletins of the Philosophical Society are offered in exchange for the publications of lenrned societies and institutions, universitiea and libraries. The Bulletins are issued at irregular intervals in the form of Beparate papers. These are made up into volumes of about 400 pages each, for each of the Humanistic, Scientitic und Medical Sections. Separate numbers may be purchused from the Registrar of the University, to whom remittances should be addreased.

PRICE, $\$ 0.75$.

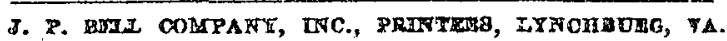




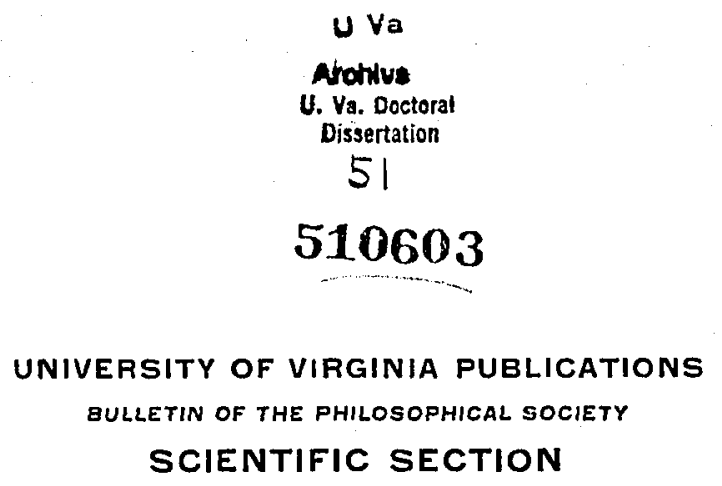

Vol. 1, No. 4; pp. $85-126$

June, 1910

\section{NEW POSITIONS. OF THE STARS IN THE HUYGHENIAN REGION OF THE GREAT NEBULA IN ORION}

From Observations made at the Leander McCormick Observatory.*

BY

RATTPH F. WITSON.

\section{CONIENIS.}

I. Ixtrowectox-

Review of previous work: Ferschel, 1834-8; W. C. Bond, 1847-8; Tassell, 1854; Thiapumofi and Struve, 1S62; G. P. Bond, 1859.65; -New stars-Precessions-Conclusions ................. 35

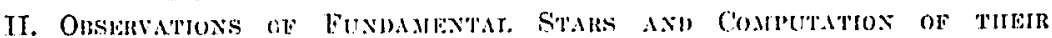

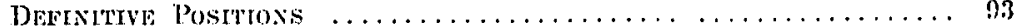

III. OUSFRntTons-

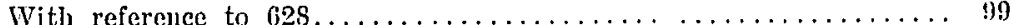

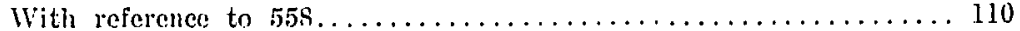

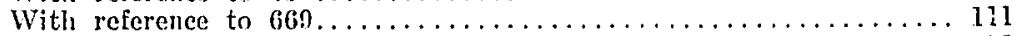

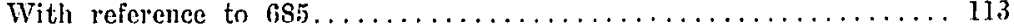

Miscellaneous $\ldots \ldots \ldots \ldots \ldots \ldots \ldots \ldots \ldots \ldots \ldots \ldots \ldots \ldots \ldots \ldots \ldots \ldots$

IV. Catalogive of Postrtons............................... 11n

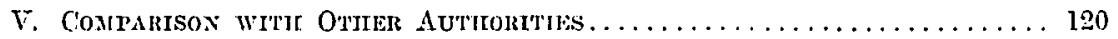

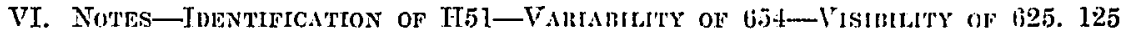

\section{PART I.}

INTRODUCTION.

The first survey of the Great Nebula in Orion for star positions was made by Sir .Tohn Ferschel at the Cape of Good Hope in the years 1834-S. This survey was made for the purpose of plotting the stars on a drawing of the nebula and included 150 stars, some of which were faint. The positions of the stars with reference to $\Theta$ Orionis were determined by eye estimates of position within the "skeleton" triangles, the vertices of which were determined by micrometrical measurement. The differences in right

*Read before the Scientific Section of the University of Virginia Philosophical Society at its meeting in May, 1910. Presented to the Fraculty of the University of Virginia in partial fulfilment of the requirements for the degree of Doctor of Philosophy. 
ascension were estimated to tenths of seconds of time and those in declination to "parts," the value of each "part" being approximately 0." 2 . Even the brighter stars were liable to an error of at least one or two of the units in which their positions were read, and a relatively large error must be expected for the fainter stars.

In the years 1847-8 Professor W. C. Bond, at Harvard College Observatory, made a series of micrometrical observations of 96 stars in the nebula. [H. C. O. Annals, Vol. V.] In most cases the relative positions of the stars are based on but one or two measures, and consequently have large probable errors.

The work of Lassell, published in the Memoirs of the Royal Astrononical Society, 1854, contains positions of 59 of the brighter stars. The position angles with reference to $\Theta$ were read to minutes and the distances to seconds of arc, so that the probable error of all the positions is quite large.

More accurate as well as more extensive were the later surveys of Liapunoff and Struve, St. Petersburg, 1862, and of G. P. Bond, at Harvard College Observatory, 1859-65 [H. C. 0. Annals, Vol. V]. The work of Professor Bond was an extensive survey of the whole nelunla to determine the positions of all the stars visible in the 15-inch equatorial of the Harvard Observatory. The catalogue contains the positions, relative to $\Theta$ Orionis, of 1101 stars, down to approximately the fouiteenth magnitude. The positions were for the most part determined by transits for right ascension and mica scale readings for declination. In the cases of the fainter stars, however, these mothods were found impracticable and the positions of a large number of these stars were determined from diagrams. In some cases the diagrams were supplemented by one or two micrometer measures, but for all the faint stars there is reason to suspect an appreciable probable error. I was especially surprised to note the number of such determinations in the Fuyghenian region of the nebula. Out of 51 stars assigned to this region by Professor E. S. Holden in his "Monograph on the Central Positions of the Nebula in Orion," the positions of eighteen were determined by means of diagrams, while those of eight others were determined from measures with reference to auxiliary stars, the positions of which were no more accurately determined than those of the average of the brighter stars.

The positions of 47 of the bright stars in the nebula were determined heliometrically by Bruno Meyermann at Groningen in 1905.' Considering the large probable errors of former work on the faint stars, and 
having placed at $m y$ disposal the 26-inch refractor of the Leander MCCormick Observatory, it seemed desirable to apply direct micrometrical measurements to these stars in the Fuyghenian region in sufficient numbers to obtain fairly accurate results. It further seemed probable: 1, that some of the brighter stars, the former positions of which ought to be fairly accurate, might in fifty or more years show some proper motion independent of that of the trapezium; 2, that, during the same period of time, new variable stars might have appeared; 3 , that some stars fainter than those catalogued by Professor Bond might be seen with a telescope of larger aperture.

The observations were begun in September, 1908, and cover a period of eighteen months. All the observations were made in position-angle and distance. With the vicw to decreasing the errors arising from the measurement of long distances, I adopted as fundamental stars 628 ( $\Theta$ Orionis) and 558,669 , and 685 , stars forming a triangle about 628 . These numbers are the number's of the stars in Bond's catalogue [H. C. O. Annals, Vol. V], and his designations are used throughout this paper, except in the cases of stars which he did not catalogue. The position-angle of all six possible combinations of the fundamental stars were measured on forty nights. The measures of distances of the same combinations would average about three less. From the average of the obscrved position-angles and distances, differences in right ascension and declination were determined, and from these the final differential positions with reference to 628 were determined by a least square solution. "The positions of the stars within 100 " of the trapezium were determined by measures with reference to 628 directly. The positions of all stars outside of this central group were measured. with reference to the nearest fundamental star and through it referred to 628 . In some cases exceptions were made to this plan and two sets of measures were made, one with reference to the nearest fundamental star, the other with reference to 628 direct. In such cases both sets have been reduced to differences in right ascension and declination. The first set has then been referred to 628 by means of the definitive position of the fundamental star and the two sets have then been combined, giving each observation equal weight, to form the final position relative to 628. As far as practicable, measures of position-angle and distance were made on ten nights for each star. This plan has been carried out on all but five of the Bond stars and none of these five, save 625, has been observed on less than seven nights. See note on 625 in Part VI. 
The pitch of the micrometer screw was detcrmined at different temperatures by transits of polar and equatorial stars and by measures of differences in right ascension of stars in the Pleiades. The observed and alopted values of the pitch are given in the following bable:

$\begin{array}{ccc}\text { Temperature. } & \text { Observed. } & \text {-1dopted. } \\ 70^{\circ} & 9 . " \mathrm{~S} 62 & 9 . " \mathrm{~S} 61 \\ 50 & .855 & .856 \\ 30 & .85 \% & .851\end{array}$

The value of the pitch of the screw lor the mean temperature for ench set of obscrvations is given by the formula-

$$
\mathrm{P}=9 . " \text {. } 850-0 . " 00025\left(50^{\circ}-\mathrm{t}\right) \text {. }
$$

The values derived from this formula were used for the fundamental stars. For the other stars the mean value $9 . " 856$ was nsed, the errors arising therefrom being negligible in comparison with the errors of obscrvation.

On the best nights it was my custom to observe the fainter stars and to look for other's not catalogued by Professor Bond. Through this search I found five stars, the existence of which is certain. I do not believe that these stars have been catalogued before, and make a special note of their positions in the hope that some one may be interested enough to look them up.

$\begin{array}{crrc} & \Delta a & \Delta \delta & \text { Nights. } \\ 1 . & -86 .{ }^{\prime \prime} 6 & +89 . " 5 & 4 \\ .2 . & 62.6 & -24.5 & 4 \\ 3 . & 40.6 & +7 \% .3 & 2 \\ 4 . & 23.7 & +43.8 & 10 \\ 5 . & -22.3 & +84.7 & 3\end{array}$

No. 2 is certainly variable. It was not seen at all during the first year of obscrvation, but on November' 10,11 , and 12, 1909, it was nearly as bright as 589, from which it is distant about $6^{\prime \prime}$. Upon February 1, 1910, it was considerably fainter. No. 4 must also be variable, since during the fall of 1909 it was almost as bright as 612 and $61 \mathrm{~s}$ and considerably brighter than several of the Bond stars in the region. Upon February 1 , 1910 , it also seemed to be much fainter. If either No. 2 or No. 4 had attained a maximum during the period covered by Professor Bond's observations, it is hardly probable that they would have been overlooked by him. The other three stars are very faint and would hardly have been seen by Professor Bond. 
In addition to these I obtained measures upon two other stars not catalogued by Professor Bond, the Alvan Clark star within the trapezium and a companion star to Bond 642 which is given by Professor 0 . Stone in his work on the Nobula in Orion. [Leander McCormick Obs. Publ., Vol. I, Pt. \%.]

The following will serve to explain the tabulation of the observations in Parts II and IIII:

Column 1. Dates.

2. Secing on il scerle of $\overline{0}$.

3. Mean temperature of the series of olsservations on the date given.

4. Position-angle.

5. Number of settings in position-angle.

6. Residuals in position-angle.

\%. Distance in revolutions of the micrometer screw.

S. Number of settings in distance.

9. Residuals in distance:

In Part II are given the observations on the fundamental stars, followed by the solution for their definitive positions. In Part IIT are given the observatious of the remaining stars, arranged in sets according to the fundamental stars with reference to which the measures were made. After these are added a few miscellancous measures, most of which were made before the general plan of work was mapped ont. These have been. given small weight and nsed in determining the final positions of the stars.

The catalogue of positions given in Part IV will be explained by the following:

Column 1. Number.

2. G. P. Bond's number or other designation, in case the star was not catalogued by Professor Bond.

3. Herschel and Struve number.

4. Herschel's letter.

5. W. C. Bond's number.

6. Lassell's designation.

\%. Liapunofi's letter.

8. Magnitude. [L. McCormick Publ., Vol. I, Pt. 7.]

9. Position in right ascension relative to $\Theta$ Orionis.

10. Position in declination relative to $\Theta$ Orionis.

11. Number of nights observed. 
In order to obtain comparisons between my results and those of former observers, all the positions were reduced for precession and proper motion to 1910.0. The proper motion of $\Theta$ was taken from Newcomb's Fundamental Catalogue. In computing his precession coefficients Professor Bond used Mädler's proper motion of $₫$, which is erroncous by at least $2^{\prime \prime}$ a century, so that the use of his coefficients for reducing observations separated by any considerable period of time will result in a large error.

The precessions for Part $V$ were computed by means of the formulæ, $\frac{\mathrm{d}\left(a^{\prime}-a\right)}{\mathrm{dt}}=n\left\{\sin a^{\prime}\left(\tan \delta^{\prime}-\tan \delta\right)+2 \sin \frac{1}{2}\left(a^{\prime}-a\right) \cos \frac{1}{2}\left(a^{\prime}+a\right) \tan \delta^{\prime}\right.$, $\frac{\mathrm{d}\left(\delta^{\prime}-\delta\right)}{\mathrm{dt}}=n\left(\cos a^{\prime}-\cos a\right)$;

These may be written,

$\frac{\mathrm{d}\left(a^{\prime}-a\right)}{\mathrm{dt}}=\left\{\frac{\sin a^{\prime}}{\cos \delta \cos \delta^{\prime}} \sin \left(\delta^{\prime}-\delta\right)+2 \sin \frac{1}{2}\left(a^{\prime}-a\right) \cos \frac{1}{2}\left(a^{\prime}+a\right) \tan \delta\right\}$, $\frac{d\left(\delta^{\prime}-\delta\right)}{d t}=2 n \sin \frac{1}{2}\left(a^{\prime}+a\right) \sin \frac{1}{2}\left(a^{\prime}-a\right)$.

Taking into consideration the small relative distances of the stars, we may simplify the formulæ by putting $\sin a^{\prime}=\sin a$,

$$
\begin{aligned}
& \frac{1}{2}\left(a^{\prime}+a\right)=a, \text { and } \cos \delta=\cos \delta^{\prime}, \text { whence } \\
& \frac{\mathrm{d}\left(a^{\prime}-a\right)}{\mathrm{dt}}=n\left\{\mathrm{P} \sin \left(\delta^{\prime}-\delta\right)+\mathrm{Q} \sin \frac{1}{2}\left(a^{\prime}-a\right)\right\}, \\
& \frac{\mathrm{d}\left(\delta^{\prime}-\delta\right)}{\mathrm{dt}}=R \sin \frac{1}{2}\left(a^{\prime}-a\right),
\end{aligned}
$$

where we have put $\mathrm{P}=\frac{\sin a}{\cos ^{2} \delta}, \mathrm{Q}=2 \cos a \tan \delta$, and $\mathrm{R}=2 n \sin a$.

Newcomb's value of $n, 20 . " 069$, was used, and the position of (a) for 18830, the mean of the epoch of Professor Bond's observations and my own, was taken from Newcomb's Fundamental Catalogue. The following tables of precessions were thus computed: 


\begin{tabular}{rrrrrr}
\multicolumn{5}{c}{ p. for Da } & \multicolumn{1}{c}{} \\
$\Delta \alpha$ & $\Delta \delta \pm 30^{\prime \prime}$ & $60^{\prime \prime}$ & $100^{\prime \prime}$ & $150^{\prime \prime}$ & \multicolumn{1}{c}{$200^{\prime \prime}$} \\
$\pm 30^{\prime \prime}$ & $\pm 0 . " 00295$ & \pm 0.00588 & \pm 0.00976 & \pm 0.01462 & \pm 0.01949 \\
60 & 299 & 592 & 980 & 1466 & 1953 \\
100 & 303 & 596 & 985 & 1470 & 1957 \\
150 & 309 & 602 & 991 & 1476 & 1963 \\
200 & 315 & 608 & 997 & 1482 & 1969 \\
$\mp 30$ & 287 & 575 & 960 & 1440 & 1921 \\
60 & 283 & 570 & 956 & 1436 & 1917 \\
100 & 280 & 566 & 952 & 1432 & 1913 \\
150 & 276 & 562 & 948 & 1428 & 1909 \\
200 & \pm 0.00272 & \pm 0.00558 & $\pm 0.00944 \pm 0.01424$ & \pm 0.01905
\end{tabular}

$$
\begin{array}{rr}
\multicolumn{2}{c}{\text { p. for D } \delta} \\
\Delta \alpha \\
\pm 30^{\prime \prime} & \pm 0.00289 \\
60 & 578 \\
100 & 964 \\
150 & 1445 \\
200 & \pm 0.01927
\end{array}
$$

The values of former observations reduced to 1910.0 are given in Part V. Below each set is given the mean of the results obtained by using the following weights:

H. Herschel $\ldots \ldots \ldots \ldots \ldots \ldots \ldots \ldots \ldots \ldots$

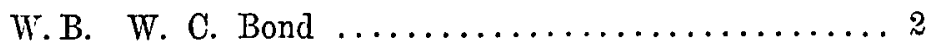

Ll. Lassell $\ldots \ldots \ldots \ldots \ldots \ldots \ldots \ldots \ldots \ldots \ldots$

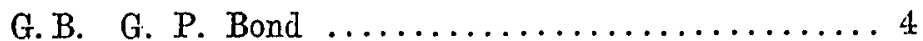

L. S. Liapunoff-Struve $\ldots \ldots \ldots \ldots \ldots \ldots \ldots \ldots \ldots$

N. Newcomb ..................... 4

A. G. C. Astronomische Gesellschoft $\ldots \ldots \ldots \ldots \ldots \ldots 4$

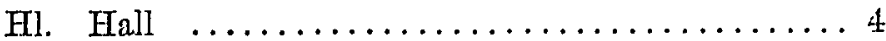

B. Burnham $\ldots \ldots \ldots \ldots \ldots \ldots \ldots \ldots \ldots \ldots$

M. Meyermann $\ldots \ldots \ldots \ldots \ldots \ldots \ldots \ldots \ldots \ldots 4$

Below the mean is given my position for each star for the sake of comparison. The comparisons with the weighted means and with the Bond positions are given in the last table. An examination of these results seems to show no evidence of any proper motion in the great majority of the stars. Almost without exception the largest residuals occur in the case of those stars, Bond's positions of which were determined by eye estimates and diagrams, and which are therefore liable to the largest 
errors. Six of the stars, however, show differences which, I do not think. arise from this cause. They are 612,618,622,636, 686, and 688, and may be studied in pairs. 612 and 618 are a pair of stars, just below the trapezium, of sufficient brightness to permit Profcssor Bond to make two measures of position in addition to several diagrams. The differences in the case of these two stars would seem to indicate a northward proper motion in declination of between $4 "$ and $5 "$ a century.

The differences in right ascension in the cases of 622 and 636 can be largely explained by assuming that these stars are physically connected with the trapezium and share in its motion. This assumption is not improbable, as both the stars are within $30^{\prime \prime}$ of the trapezinm. The residuals in right ascension would then reduce from

$-1 . " 0$ to $0 . " 2$ in the case of 622, and

-1.3 to 0.5 in the case of 636 .

There still remain, however, small residuals in declination which cannot be explained in this way, and there is need for more accurate measures upon which to base a discussion of this motion.

The cases of 686 and 688 are peculiar. The only basis of comparison in each case is a single estimate of position given by Prof. Bond, from which the following residuals were obtained:

$$
\begin{array}{rrr} 
& \Delta \Delta a & \Delta \Delta \delta \\
686 & +2^{\prime \prime} .6 & +22^{\prime \prime} .0 \\
688 & -23.8 & 4.6
\end{array}
$$

Evidently either something is wrong or these stars have large proper motion. The stars themselves are distinctly visible with the 26-inch equatorial on any niglt with the sceing 4 on a scale of 5 . There are no other stars in the immediate vicinity except 671 and 676 , and certainly none in the positions given by Professor Bond. These must be the stars which he has catalogued, but unless they are affected by large proper motions, Professor Bond's positions must be erroneous. It may be of interest ten years hence to measure the positions of these two stars in order to see if any great change of position is indicated.

Part VI contains a few notes which may be of interest to any one who is working on the Nebula of Orion.

I wish to thank Professor Stone for the use of the Jcander McCormick equatorial and for the many helpful suggestions which he has given me throughout this work.

RatPH E. WILson. 
NEW POSITIONS OF STARS IN ORION

PAR'I II.

Obscrvations of the Fundamental Stars.

\begin{tabular}{l|ll|l|ll|l|l|l}
$\mathrm{DATS}$ & $\mathrm{S}$ & $\mathrm{T}$ & $\mathrm{P}$ & $\mathrm{n}$ & $\mathrm{N}$ & $\mathrm{R}$ & $\mathrm{n}$
\end{tabular}

628-685

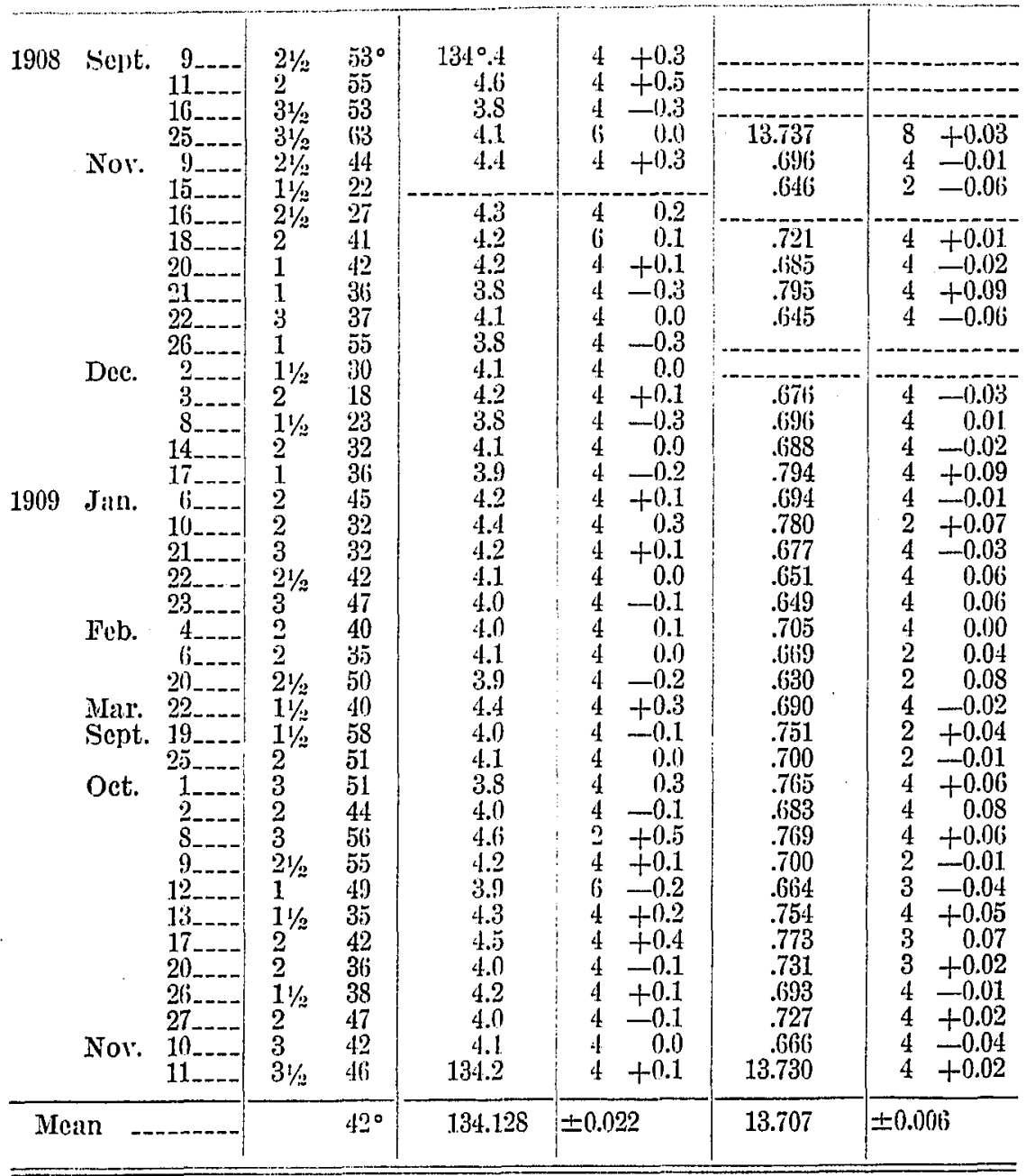


Observations of the Fundamental Stars-Continued.

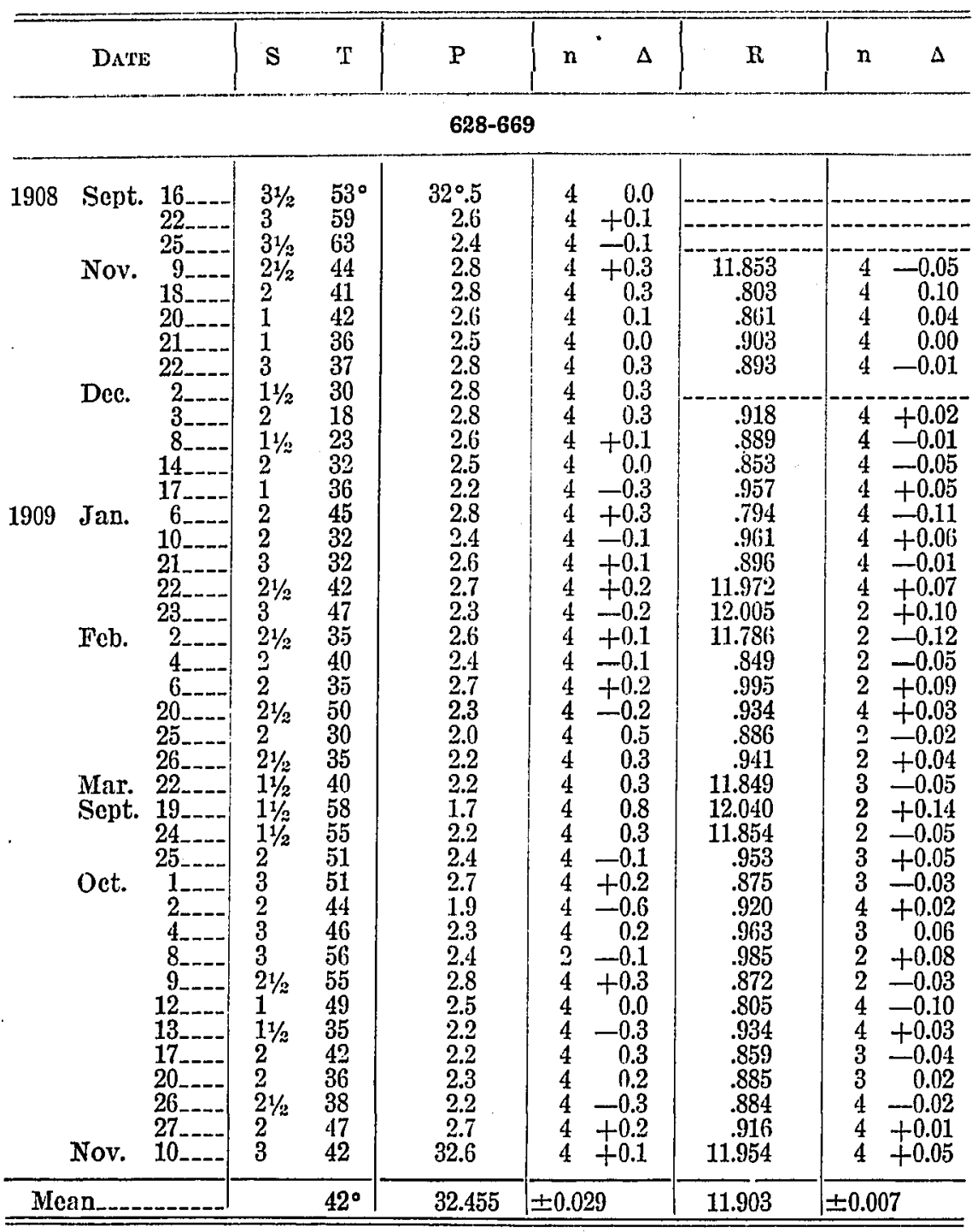


Obscrvations of the Fundamental Stars-Continued.

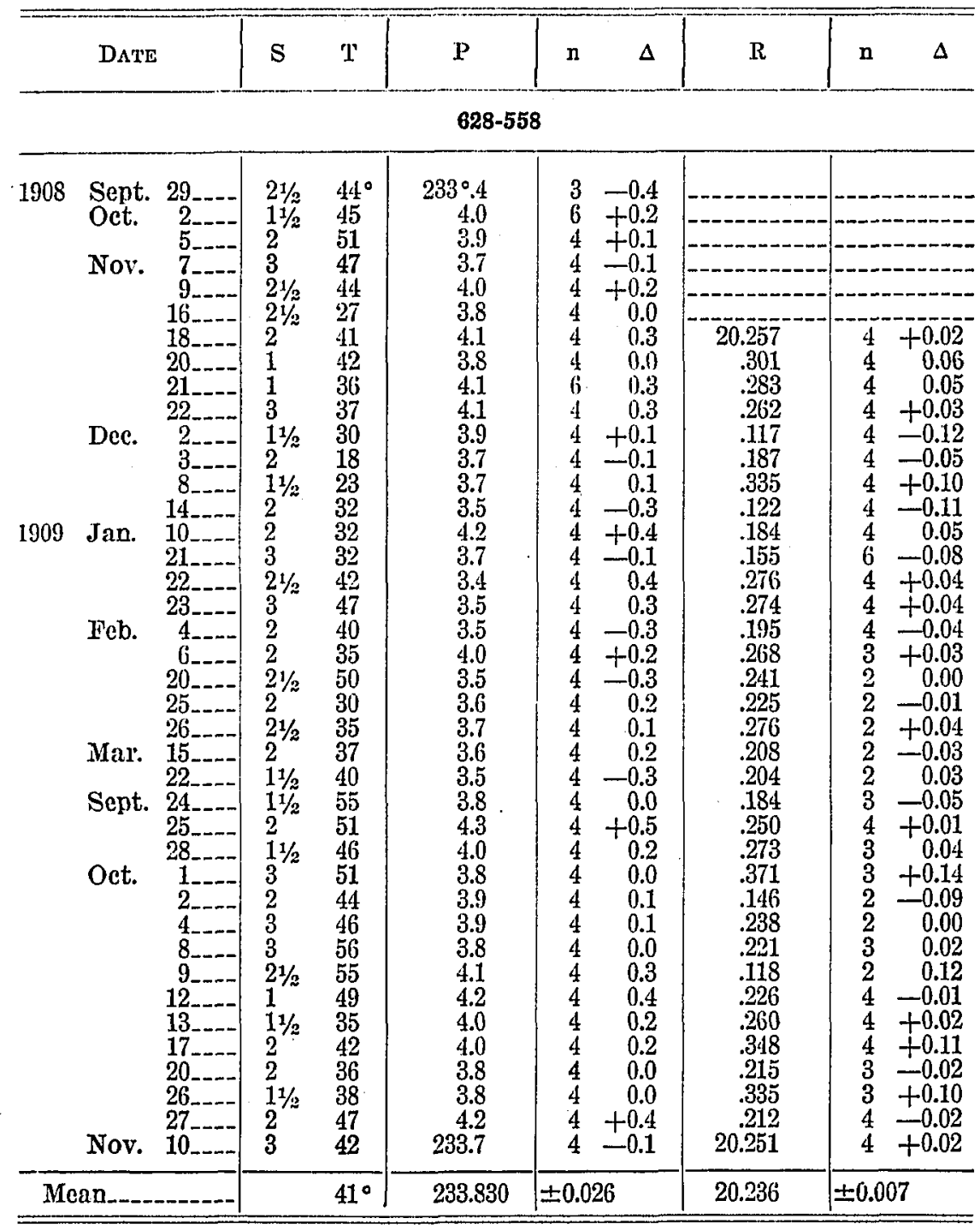


Ouservations of the Fundamental Stars-Continued.

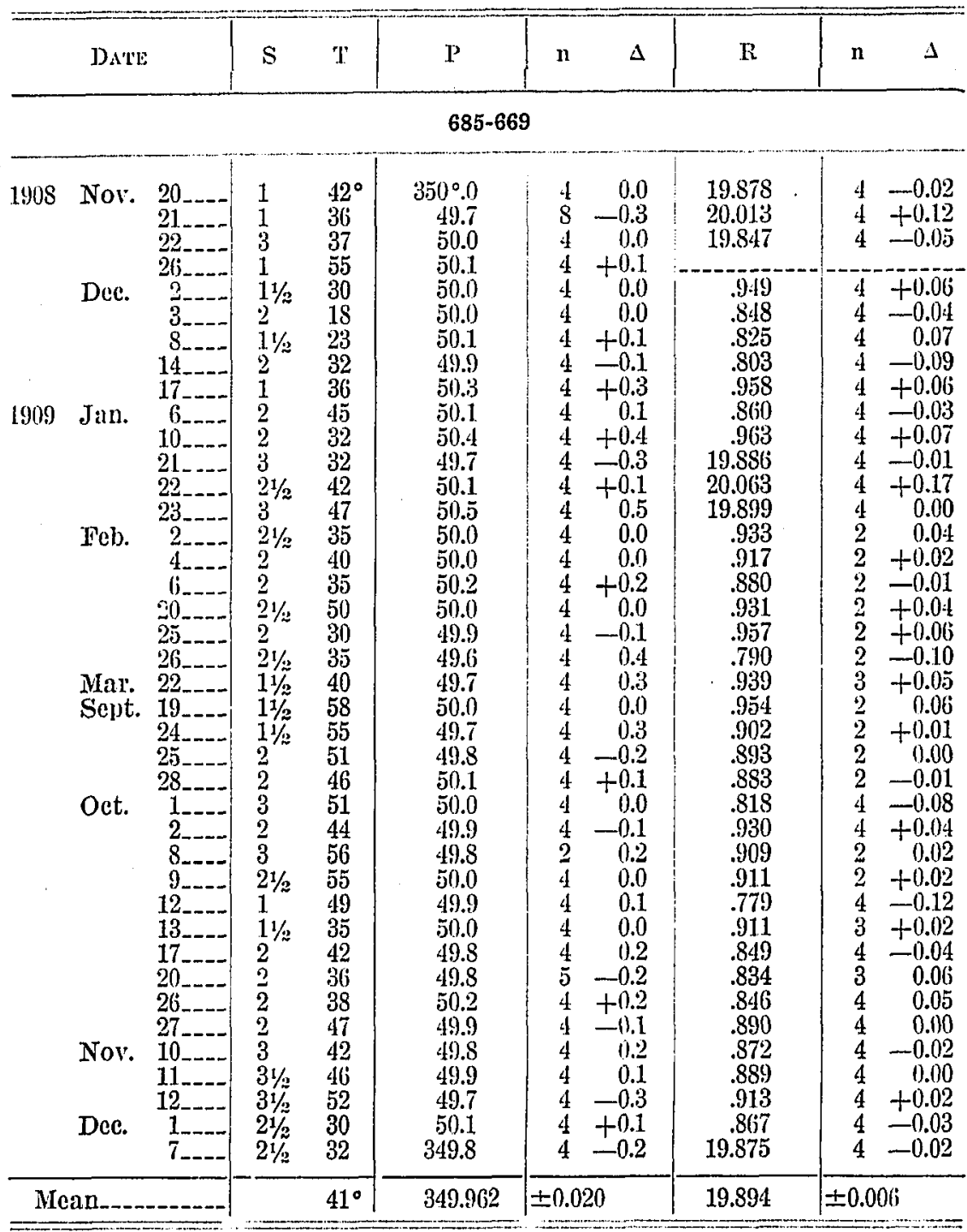


Obsertations of the Fundamental stars-Continued.

\begin{tabular}{|c|c|c|c|c|c|c|c|c|c|c|}
\hline & DATE & & $\mathrm{S}$ & 't' & $P$ & $n$ & $\Delta$ & $\mathrm{R}$ & $\mathrm{n}$ & $\Delta$ \\
\hline \multicolumn{11}{|c|}{$685-558$} \\
\hline 1909 & $\begin{array}{l}\text { Nor: } \\
\text { Dec. }\end{array}$ & 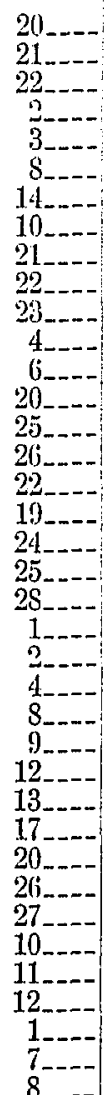 & $\begin{array}{l}1 \\
1 \\
3 \\
11 / 2 \\
2 \\
11 / 2 \\
2 \\
2 \\
3 \\
21 / 2 \\
3 \\
2 \\
2 \\
3 \\
2 \\
21 / 2 \\
11 / 2 \\
11 / 2 \\
11 / 2 \\
2 \\
11 / 2 \\
3 \\
2 \\
3 \\
3 \\
21 / 2 \\
1 \\
11 / 2 \\
2 \\
2 \\
11 / 2 \\
2 \\
3 \\
21 / 2 \\
31 / 2 \\
21 / 2 \\
21 / 2 \\
1\end{array}$ & $\begin{array}{l}42^{\circ} \\
36 \\
37 \\
30 \\
18 \\
23 \\
39 \\
32 \\
32 \\
32 \\
42 \\
47 \\
40 \\
35 \\
30 \\
30 \\
35 \\
40 \\
58 \\
55 \\
51 \\
46 \\
51 \\
44 \\
46 \\
56 \\
55 \\
49 \\
35 \\
42 \\
36 \\
38 \\
47 \\
42 \\
46 \\
52 \\
30 \\
32 \\
20\end{array}$ & $\begin{array}{r}26-1.7 \\
4.4 \\
4.8 \\
4.7 \\
4.6 \\
4.6 \\
4.4 \\
4.9 \\
4.7 \\
4.4 \\
4.17 \\
5.0 \\
4.8 \\
4.6 \\
4.5 \\
4.8 \\
4.4 \\
4.9 \\
4.7 \\
4.8 \\
4.3 \\
5.0 \\
4.8 \\
5.0 \\
4.8 \\
4.9 \\
4.7 \\
4.7 \\
4.7 \\
4.6 \\
4.4 \\
4.9 \\
4.9 \\
5.2 \\
5.3 \\
4.4 \\
4.8 \\
4.7\end{array}$ & $\begin{array}{l}4 \\
4 \\
4 \\
4 \\
4 \\
4 \\
4 \\
4 \\
4 \\
4 \\
4 \\
4 \\
4 \\
4 \\
4 \\
4 \\
4 \\
4 \\
4 \\
4 \\
4 \\
4 \\
4 \\
4 \\
2 \\
4 \\
4 \\
4 \\
4 \\
4 \\
4 \\
4 \\
4 \\
4 \\
4 \\
4 \\
4 \\
4\end{array}$ & $\begin{array}{r}0.0 \\
-0.3 \\
+0.1 \\
0.0 \\
-0.1 \\
0.1 \\
-0.3 \\
+0.2 \\
0.0 \\
-0.3 \\
-0.1 \\
+0.3 \\
+0.1 \\
-0.1 \\
-0.2 \\
+0.1 \\
+-0.3 \\
+0.2 \\
0.0 \\
+0.1 \\
-0.1 \\
+0.3 \\
0.1 \\
0.3 \\
0.1 \\
+0.2 \\
0.0 \\
0.0 \\
0.0 \\
-0.1 \\
-0.3 \\
+0.2 \\
0.2 \\
0.5 \\
+0.6 \\
-0.3 \\
+0.1 \\
0.0\end{array}$ & $\begin{array}{r}26.280 \\
.385 \\
.233 \\
.379 \\
.137 \\
.207 \\
.157 \\
.273 \\
.196 \\
.371 \\
.423 \\
.230 \\
.331 \\
.190 \\
.214 \\
.204 \\
.361 \\
.372 \\
.151 \\
.391 \\
.393 \\
.375 \\
.419 \\
.317 \\
.245 \\
.176 \\
.197 \\
.196 \\
.207 \\
.205 \\
.215 \\
.259 \\
.219 \\
.347 \\
.269 \\
.168 \\
.248\end{array}$ & $\begin{array}{l}4 \\
4 \\
4 \\
4 \\
4 \\
4 \\
4 \\
4 \\
4 \\
4 \\
4 \\
2 \\
2 \\
4 \\
3 \\
4 \\
3 \\
3 \\
2 \\
2 \\
2 \\
3 \\
4 \\
3 \\
4 \\
4 \\
3 \\
4 \\
4 \\
4 \\
4 \\
4 \\
3 \\
4 \\
4 \\
4 \\
4\end{array}$ & $\begin{array}{r}+0.01 \\
+0.12 \\
+0.04 \\
+0.11 \\
-0.13 \\
0.06 \\
0.11 \\
+0.01 \\
-0.07 \\
+0.10 \\
+0.15 \\
-0.04 \\
+0.06 \\
-0.08 \\
0.06 \\
-0.07 \\
+0.09 \\
+0.10 \\
-0.12 \\
+0.12 \\
0.12 \\
0.10 \\
0.15 \\
+0.05 \\
-0.02 \\
0.09 \\
0.07 \\
0.07 \\
0.06 \\
0.06 \\
0.05 \\
0.01 \\
-0.05 \\
+0.08 \\
-0.01 \\
0.10 \\
0.02\end{array}$ \\
\hline 1910 & $\begin{array}{l}\text { Jan. } \\
\text { Feb. }\end{array}$ & $\begin{array}{r}10 \\
1\end{array}$ & $\frac{2}{3} 1 / 2$ & $\begin{array}{l}25 \\
33\end{array}$ & $\begin{array}{r}5.2 \\
264.9\end{array}$ & 4 & $\begin{array}{r}0.5 \\
+0.2\end{array}$ & 26.226 & $\begin{array}{l}4 \\
4\end{array}$ & $\begin{array}{r}0.04 \\
-0.04\end{array}$ \\
\hline \multicolumn{3}{|c|}{ Mcan } & & $40^{\circ}$ & $264^{\circ} .738$ & \pm 0.02 & & 26.267 & \pm 0.0 & \\
\hline
\end{tabular}


Observations of the Frundamental Stars-Continued.

\begin{tabular}{|c|c|c|c|c|c|c|c|c|c|c|}
\hline \multicolumn{3}{|c|}{ DAte } & $\mathbf{S}$ & $\mathrm{T}$ & $\mathrm{P}$ & $\mathbf{n}$ & $\Delta$ & $\mathrm{R}$ & $\mathbf{n}$ & $\Delta$ \\
\hline \multicolumn{11}{|c|}{$669-558$} \\
\hline 1910 & $\begin{array}{l}\text { Nov. } \\
\text { Dec. }\end{array}$ & $\begin{array}{r}20 \\
21 \\
22 \\
2 \\
3 \\
8 \\
14 \\
17 \\
10 \\
21 \\
22 \\
23 \\
4 \\
6 \\
20 \\
25 \\
26 \\
22 \\
24 \\
25 \\
28 \\
1 \\
2 \\
2 \\
4 \\
8 \\
9 \\
12 \\
13 \\
17 \\
20 \\
26 \\
27 \\
10 \\
11 \\
12 \\
12 \\
1 \\
7 \\
8 \\
10 \\
1\end{array}$ & $\begin{array}{l}1 \\
1 \\
3 \\
11 / 2 \\
2 \\
11 / 2 \\
2 \\
1 \\
2 \\
3 \\
21 / 2 \\
3 \\
2 \\
2 \\
21 / 2 \\
2 \\
21 / 2 \\
11 / 2 \\
11 / 2 \\
2 \\
11 / 2 \\
3 \\
2 \\
3 \\
3 \\
21 / 2 \\
11 / 2 \\
2 \\
2 \\
2 \\
11 / 2 \\
2 \\
3 \\
31 / 2 \\
31 / 2 \\
21 / 2 \\
21 / 2 \\
1 \\
21 / 2 \\
3\end{array}$ & $\begin{array}{l}42^{\circ} \\
36 \\
37 \\
30 \\
18 \\
23 \\
32 \\
36 \\
32 \\
32 \\
42 \\
47 \\
40 \\
35 \\
50 \\
30 \\
35 \\
40 \\
55 \\
51 \\
46 \\
51 \\
44 \\
46 \\
56 \\
55 \\
49 \\
35 \\
42 \\
30 \\
38 \\
47 \\
42 \\
46 \\
52 \\
30 \\
32 \\
29 \\
25 \\
33\end{array}$ & $\begin{array}{r}226^{\circ} .1 \\
5.6 \\
5.7 \\
6.0 \\
5.9 \\
5.7 \\
5.8 \\
5.6 \\
6.1 \\
5.9 \\
5.8 \\
6.0 \\
5.8 \\
5.7 \\
5.4 \\
5.5 \\
5.5 \\
5.4 \\
5.9 \\
5.8 \\
6.0 \\
5.8 \\
5.7 \\
5.9 \\
5.6 \\
6.0 \\
5.8 \\
6.1 \\
6.0 \\
5.8 \\
6.0 \\
6.1 \\
5.9 \\
5.8 \\
6.1 \\
5.9 \\
6.1 \\
5.6 \\
6.2 \\
226.2\end{array}$ & 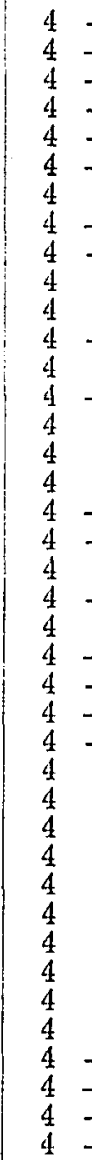 & $\begin{array}{r}+0.3 \\
-0.2 \\
-0.1 \\
+0.2 \\
+0.1 \\
+0.1 \\
0.0 \\
-0.2 \\
+0.3 \\
0.1 \\
0.0 \\
+0.2 \\
0.0 \\
-0.1 \\
0.4 \\
0.3 \\
0.3 \\
-0.4 \\
+0.1 \\
0.0 \\
+0.2 \\
0.0 \\
-0.1 \\
+0.1 \\
-0.2 \\
+0.2 \\
0.0 \\
0.3 \\
0.2 \\
0.0 \\
0.2 \\
0.3 \\
0.1 \\
0.0 \\
0.3 \\
0.1 \\
+0.3 \\
-0.2 \\
+0.4 \\
+0.4\end{array}$ & $\begin{array}{r}31.526 \\
.853 \\
.633 \\
.611 \\
.558 \\
.621 \\
.537 \\
.888 \\
.572 \\
.671 \\
.764 \\
.613 \\
.604 \\
.723 \\
.539 \\
.635 \\
.692 \\
.584 \\
.561 \\
.693 \\
.680 \\
.695 \\
.728 \\
.730 \\
.652 \\
.667 \\
.613 \\
.671 \\
.675 \\
.520 \\
.688 \\
.715 \\
.726 \\
.729 \\
.675 \\
.546 \\
.581 \\
.702 \\
.582 \\
31.653\end{array}$ & $\begin{array}{l}4 \\
4 \\
4 \\
4 \\
4 \\
4 \\
4 \\
4 \\
4 \\
4 \\
4 \\
4 \\
2 \\
4 \\
4 \\
4 \\
4 \\
4 \\
3 \\
2 \\
2 \\
3 \\
4 \\
3 \\
3 \\
3 \\
4 \\
4 \\
4 \\
4 \\
4 \\
4 \\
4 \\
4 \\
4 \\
4 \\
4 \\
2 \\
4 \\
4\end{array}$ & $\begin{array}{r}-0.12 \\
+0.20 \\
-0.02 \\
0.04 \\
0.09 \\
0.03 \\
-0.11 \\
+0.24 \\
0.08 \\
+0.02 \\
+0.11 \\
-0.04 \\
-0.05 \\
+0.07 \\
-0.11 \\
-0.02 \\
+0.04 \\
-0.07 \\
0.09 \\
+0.04 \\
0.03 \\
0.04 \\
0.08 \\
0.08 \\
0.00 \\
+0.01 \\
0.04 \\
+0.02 \\
+0.02 \\
0.13 \\
+0.04 \\
0.06 \\
0.07 \\
0.08 \\
+0.02 \\
-0.10 \\
0.07 \\
+0.05 \\
-0.07 \\
0.00\end{array}$ \\
\hline Mc & $a n$ & $=$ & & $40^{\circ}$ & 225.838 & \pm 0.024 & & 31.653 & \pm 0. & \\
\hline
\end{tabular}


The reduction of the mean position-angles and distances for the fundamental stars to differences in right ascension and declination gives the following results:
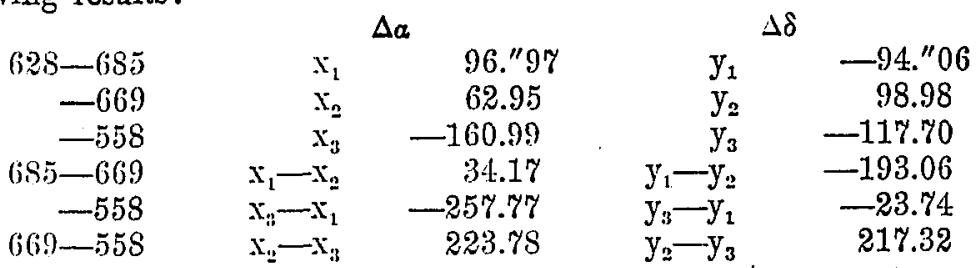

A least square solution of these twelve equations gives as definitive positions with reference to 628 ,

$\begin{array}{ccc} & \Delta a & \\ 655 & 96 . " 96 & -94 . .^{\prime \prime} 04 \\ 669 & 62.87 & 99.14 \\ 558 & -160.91 & -117.8^{77}\end{array}$

PART III.

Observations with reference to 628 .

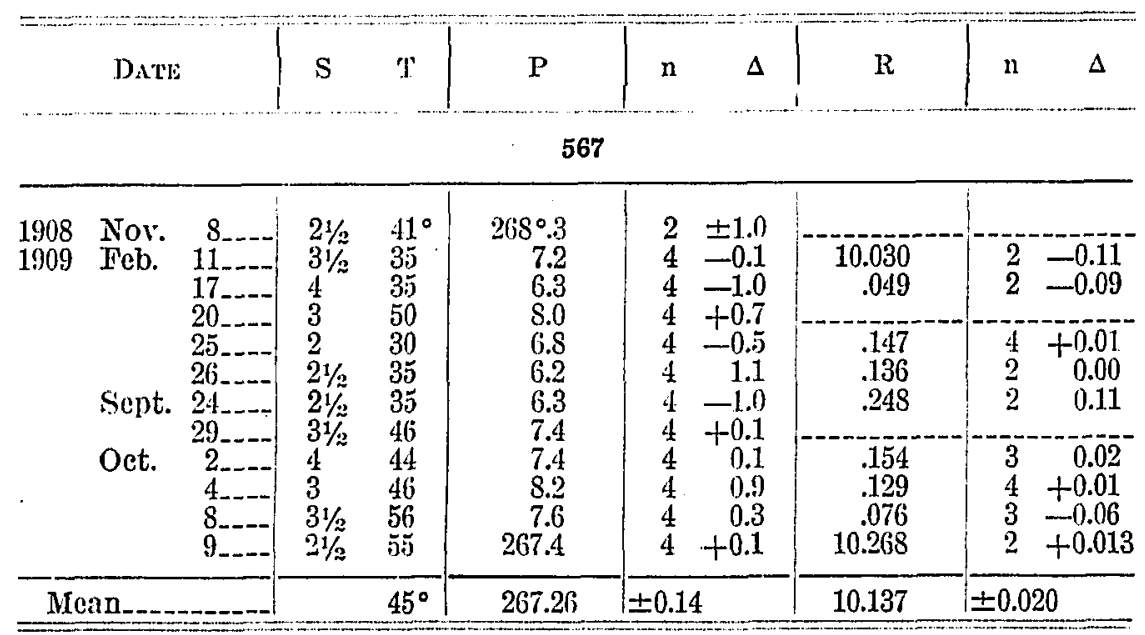


Obsercations with refercuce to 628-Continted.

\begin{tabular}{|c|c|c|c|c|c|c|c|c|c|c|}
\hline & DATE & & $s$ & $\mathrm{~T}$ & $P$ & $n$ & $\Delta$ & $\mathrm{R}$ & n & $\Delta$ \\
\hline \multicolumn{11}{|c|}{575} \\
\hline 1909 & $\begin{array}{l}\text { Nov. } \\
\text { Dec. } \\
\text { Jan. } \\
\text { Fob. } \\
\text { Mar. }\end{array}$ & $\begin{array}{r}16 \\
18 \\
22 \\
3 \\
3 \\
21 \\
23 \\
23 \\
2 \\
6 \\
6 \\
20 \\
20 \\
22\end{array}$ & $\begin{array}{l}21 / 2 \\
2 \\
3 \\
2 \\
3 \\
3 \\
21 / 2 \\
2 \\
3 \\
11 / 2\end{array}$ & $\begin{array}{l}27^{\circ} \\
41 \\
37 \\
18 \\
32 \\
47 \\
35 \\
35 \\
50 \\
40\end{array}$ & $\begin{array}{r}255 \circ .9 \\
\quad(6.0 \\
5.1 \\
5.4 \\
5.4 \\
5.5 \\
5.4 \\
5.1 \\
5.4 \\
4.9 \\
254.9\end{array}$ & $\begin{array}{l}4 \\
4 \\
4 \\
4 \\
4 \\
4 \\
6 \\
4 \\
4 \\
4\end{array}$ & $\begin{array}{r}+0.5 \\
+0.6 \\
-0.3 \\
0.0 \\
0.0 \\
+0.1 \\
0.0 \\
+0.2 \\
-0.5 \\
-0.5\end{array}$ & $\begin{array}{l}9.057 \\
9.020 \\
8.914 \\
8.820 \\
9.085 \\
9.015 \\
8.808 \\
9.101 \\
8.988 \\
8.931\end{array}$ & $\begin{array}{l}4 \\
2 \\
4 \\
4 \\
2 \\
2 \\
2 \\
2 \\
4 \\
3\end{array}$ & $\begin{array}{r}+0.0 \mathrm{~S} \\
+0.05 \\
-0.06 \\
-0.15 \\
+0.11 \\
+0.04 \\
+0.17 \\
+0.13 \\
+0.01 \\
-.0 .01\end{array}$ \\
\hline \multicolumn{3}{|c|}{ Mean } & & $37^{\circ}$ & 255.41 & \multicolumn{2}{|c|}{ \pm 0.07} & 8.974 & \multicolumn{2}{|c|}{ \pm 0.022} \\
\hline
\end{tabular}

589

\begin{tabular}{|c|c|c|c|c|c|c|c|c|c|}
\hline 1908 & Nor. & $16_{-\ldots-}$ & \multirow{11}{*}{$\begin{array}{l}3 \\
21 / 2 \\
3 \\
3 \\
21 / 2 \\
3 \\
2 \\
21 / 2 \\
2 \\
21 / 2 \\
3 \\
31 / 2\end{array}$} & \multirow{4}{*}{$\begin{array}{l}27^{\circ} \\
41 \\
37\end{array}$} & \multirow{3}{*}{$\begin{array}{r}250^{\circ} .0 \\
50.3 \\
50.0\end{array}$} & & \multirow{2}{*}{$\begin{array}{r}+0.1 \\
0.4\end{array}$} & & \\
\hline & & 18 & & & & & & & \\
\hline & & 22 & & & & 4 & +0.1 & 6.158 & -0.05 \\
\hline 1909 & . Tan. & 23 & & & 49.8 & 4 & -0.1 & .249 & $+0.0-t$ \\
\hline & Fob. & & & 35 & 49.6 & 4 & 0.3 & .251 & 0.05 \\
\hline & & 20 & & 50 & 49.8 & 4 & 0.1 & .402 & +0.20 \\
\hline & & $25 \ldots$ & & 30 & 49.5 & 4 & 0.4 & .130 & -0.08 \\
\hline & Ylar & 15 & & 85 & 49.5 & $\frac{4}{4}$ & -0.4 & $\begin{array}{l}.140 \\
283\end{array}$ & $\begin{array}{l}70.00 \\
+0.08\end{array}$ \\
\hline & & 17 & & 37 & 50.1 & 4 & $\begin{array}{r}+0.2 \\
+\end{array}$ & .174 & -0.03 \\
\hline & Sicpt. & 29 & & 46 & 49.9 & 4 & 0.0 & .060 & -0.14 \\
\hline & Oet. & S... & & 56 & 250.2 & & +0.3 & (6.204 & 30.00 \\
\hline Mo & $\ln$ & -..-.-- & & $40^{\circ}$ & 249.88 & \pm 0.05 & & 0.205 & \pm 0.020 \\
\hline
\end{tabular}

\begin{tabular}{|c|c|c|c|c|c|c|c|c|}
\hline 1910 & $\begin{array}{l}\text { Feb. } \\
\text { Oct. } \\
\text { Nov. } \\
\text { Feb. }\end{array}$ & 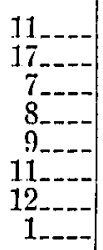 & $\begin{array}{l}31 / 2 \\
4 \\
4 \\
4 \\
21 / 2 \\
31 / 2 \\
3 \\
3\end{array}$ & $\begin{array}{l}35^{\circ} \\
35 \\
56 \\
56 \\
55 \\
46 \\
52 \\
33\end{array}$ & $\begin{array}{r}250^{\circ} .6 \\
50.2 \\
49.3 \\
49.3 \\
50.5 \\
53.2 \\
52.0 \\
249.9\end{array}$ & $\begin{array}{rr}4 & 0.0 \\
4 & -0.4 \\
4 & 1.3 \\
4 & 1.3 \\
4 & -0.1 \\
4 & +2.6 \\
4 & +1.4 \\
4 & -0.7\end{array}$ & $\begin{array}{l}5.000 \\
5.030 \\
4.950 \\
5.056 \\
4.963 \\
5.000 \\
5.074 \\
4.941\end{array}$ & $\begin{array}{rr}2 & 0.00 \\
4 & +0.03 \\
4 & -0.05 \\
4 & +0.05 \\
4 & -0.04 \\
6 & 0.00 \\
3 & +0.07 \\
4 & -0.06\end{array}$ \\
\hline $\mathrm{Mc}$ & $a n_{-}$ & & . & $46^{\circ}$ & 250.62 & \pm 0.31 & 5.002 & \pm 0.012 \\
\hline
\end{tabular}


Obscrations with reference to 628 -Continued.

\begin{tabular}{|c|c|c|c|c|c|c|c|c|c|}
\hline & DATE & & $\mathbf{S}$ & 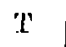 & $p$ & $n$ & $\Delta$ & $R$ & $\mathrm{n}$ \\
\hline \multicolumn{10}{|c|}{601} \\
\hline 1909 & $\begin{array}{l}\text { Feb. } \\
\text { Oct. } \\
\text { Nov. }\end{array}$ & $\begin{array}{r}11 \\
17 \\
7 \\
8-\ldots- \\
9-\ldots- \\
11-\ldots- \\
12-\ldots\end{array}$ & $\begin{array}{l}31 / 2 \\
4 \\
4 \\
4 \\
21 / 2 \\
31 / 2 \\
31 / 2\end{array}$ & $\begin{array}{l}35^{\circ} \\
35 \\
56 \\
56 \\
55 \\
46 \\
52\end{array}$ & $\begin{array}{r}227^{\circ} .8 \\
26.0 \\
30.9 \\
29.1 \\
28.8 \\
31.5 \\
230.7\end{array}$ & $\begin{array}{l}4 \\
4 \\
4 \\
6 \\
6 \\
6 \\
6 \\
4 \\
+\end{array}$ & $\begin{array}{r}-1.5 \\
-3.3 \\
+1.6 \\
-0.2 \\
-0.5 \\
+2.2 \\
+1.4\end{array}$ & $\begin{array}{r}4.460 \\
.450 \\
.277 \\
.552 \\
.288 \\
.460 \\
4.405\end{array}$ & $\begin{array}{ll}2 & +0.05 \\
2 & +0.04 \\
4 & -0.14 \\
4 & +0.14 \\
3 & -0.13 \\
4 & +0.05 \\
5 & -0.01\end{array}$ \\
\hline \multicolumn{3}{|c|}{ Mean_-_-_-_- } & & $48^{\circ}$ & 229.26 & \pm 0.53 & & 4.413 & \pm 0.028 \\
\hline \multicolumn{10}{|c|}{602} \\
\hline 1909 & $\begin{array}{l}\text { Feb. } \\
\text { Sept. } \\
\text { Oct. } \\
\text { Nov. } \\
\frac{1}{1}\end{array}$ & 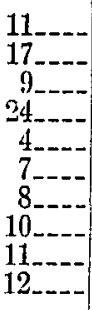 & $\begin{array}{l}31 / 2 \\
4 \\
4 \\
21 / 2 \\
3 \\
4 \\
3 \\
3 \\
31 / 2 \\
3\end{array}$ & $\begin{array}{l}35^{\circ} \\
35 \\
58 \\
55 \\
46 \\
56 \\
56 \\
42 \\
46 \\
52\end{array}$ & $\begin{array}{r}208^{\circ} .2 \\
8.3 \\
7.5 \\
7.8 \\
8.6 \\
6.4 \\
8.8 \\
8.4 \\
9.3 \\
207.8\end{array}$ & $\begin{array}{l}4+ \\
4+ \\
45 \\
4 \\
4+ \\
45 \\
4+ \\
45 \\
4+ \\
4\end{array}$ & $\begin{array}{r}+0.1 \\
+0.2 \\
+0.6 \\
-0.3 \\
+0.5 \\
-1.7 \\
+0.7 \\
0.3 \\
+1.2 \\
-0.3\end{array}$ & $\begin{array}{r}7.103 \\
.047 \\
.199 \\
.147 \\
7.043 \\
6.927 \\
7.062 \\
.172 \\
.095 \\
7.014\end{array}$ & $\begin{array}{lr}2 & +0.02 \\
2 & -0.03 \\
3 & +0.12 \\
2 & +0.07 \\
2 & -0.04 \\
4 & 0.15 \\
3 & -0.00 \\
4 & +0.09 \\
4 & +0.01 \\
4 & -0.07\end{array}$ \\
\hline \multicolumn{3}{|c|}{ Mean } & & $47^{\circ}$ & 208.07 & \pm 0.16 & & 7.081 & \pm 0.017 \\
\hline \multicolumn{10}{|c|}{608} \\
\hline 1909 & $\begin{array}{l}\text { Feb. } \\
\text { Sept. } \\
\text { Oct. } \\
\text { Nov. }\end{array}$ & 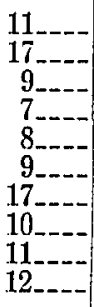 & $\begin{array}{l}31 / 2 \\
4 \\
4 \\
4 \\
4 \\
21 / 2 \\
21 / 2 \\
31 / 2 \\
31 / 2 \\
31 / 2\end{array}$ & $\begin{array}{l}35^{\circ} \\
35 \\
58 \\
56 \\
56 \\
55 \\
42 \\
42 \\
46 \\
52\end{array}$ & $\begin{array}{r}233^{\circ} .4 \\
4.9 \\
3.6 \\
7.0 \\
3.9 \\
5.0 \\
5.3 \\
7.6 \\
3.5 \\
235.5\end{array}$ & $\begin{array}{l}4 \\
4 \\
4 \\
4 \\
5 \\
5 \\
6 \\
4 \\
4 \\
6 \\
5 \\
5\end{array}$ & $\begin{array}{r}-1.6 \\
0.1 \\
-1.4 \\
+2.0 \\
-1.1 \\
0.0 \\
+0.3 \\
+2.6 \\
-1.5 \\
+0.5\end{array}$ & $\begin{array}{r}2.829 \\
.847 \\
.779 \\
.804 \\
.946 \\
2.875 \\
3.048 \\
2.801 \\
.913 \\
2.885\end{array}$ & $\begin{array}{rr}2 & -0.04 \\
4 & 0.02 \\
3 & 0.09 \\
3 & -0.07 \\
3 & +0.07 \\
3 & 0.00 \\
3 & +0.18 \\
4 & -0.07 \\
3 & +0.04 \\
4 & +0.01\end{array}$ \\
\hline \multicolumn{3}{|c|}{ Mean_........... } & & $48^{\circ}$ & 234.97 & \multicolumn{2}{|l|}{ \pm 0.30} & 2.872 & \pm 0.016 \\
\hline
\end{tabular}


Observations with reference to 628 -Continued.

\begin{tabular}{|c|c|c|c|c|c|c|c|c|c|c|}
\hline \multicolumn{3}{|c|}{ Date } & $\mathrm{S}$ & 'I' & $P$ & $\mathrm{n}$ & $\Delta$ & $\mathrm{R}$ & $\mathrm{n}$ & $\Delta$ \\
\hline \multicolumn{9}{|c|}{612} & \multicolumn{2}{|l|}{. } \\
\hline 1909 & $\begin{array}{l}\text { Jan. } \\
\text { Feb. } \\
\text { Mar. } \\
\text { Sept. } \\
\text { Oct. }\end{array}$ & $\begin{array}{r}23 \ldots \\
17 \\
20 \\
26 \\
15 \\
17 \ldots \\
23 \ldots \\
9 \\
29 \\
1 \ldots\end{array}$ & $\begin{array}{l}3 \\
4 \\
3 \\
21 / 2 \\
2 \\
21 / 2 \\
31 / 2 \\
4 \\
31 / 2 \\
3\end{array}$ & $\begin{array}{l}47^{\circ} \\
35 \\
50 \\
35 \\
37 \\
37 \\
40 \\
58 \\
46 \\
51\end{array}$ & $\begin{array}{r}326^{\circ} .4 \\
4.6 \\
5.2 \\
5.2 \\
6.1 \\
6.1 \\
4.5 \\
5.2 \\
4.9 \\
325.8\end{array}$ & $\begin{array}{l}6 \\
4 \\
4 \\
4 \\
4 \\
4 \\
4 \\
4 \\
4 \\
4\end{array}-$ & $\begin{array}{r}+1.0 \\
-0.8 \\
0.2 \\
-0.2 \\
+0.7 \\
+0.7 \\
+0.9 \\
0.2 \\
-0.5 \\
+0.4\end{array}$ & $\begin{array}{r}3.263 \\
.189 \\
.327 \\
.240 \\
.232 \\
.279 \\
.305 \\
.150 \\
.228 \\
3.254\end{array}$ & $\begin{array}{l}\frac{2}{2} \\
2 \\
4 \\
3 \\
3 \\
3 \\
2 \\
2 \\
2\end{array}$ & $\begin{array}{l}+0.02 \\
-0.06 \\
+0.08 \\
-0.01 \\
-0.02 \\
+0.03 \\
+0.06 \\
-0.10 \\
-0.02 \\
+0.01\end{array}$ \\
\hline \multicolumn{3}{|c|}{ Mean } & & $44^{\circ}$ & 325.40 & \multicolumn{2}{|l|}{ \pm 0.15} & 3.247 & \multicolumn{2}{|c|}{ \pm 0.011} \\
\hline
\end{tabular}

617

\begin{tabular}{|c|c|c|c|c|c|c|c|c|c|c|}
\hline 1908 & Scpt. & & $21 / 2$ & $53^{\circ}$ & $321^{\circ} .2$ & 6 & -0.4 & $1.696 \mathrm{i}$ & 4 & +0.01 \\
\hline & & $\begin{array}{l}16- \\
22\end{array}$ & $\begin{array}{l}31 / 2 \\
3\end{array}$ & $\begin{array}{l}53 \\
59\end{array}$ & $\begin{array}{l}2.2 \\
2.6\end{array}$ & $\begin{array}{l}4 \\
4\end{array}$ & $\begin{array}{l}+10.6 \\
+1.0\end{array}$ & .655 & 4 & $-0,03$ \\
\hline & & & $31 \%$ & 63 & 0.9 & 4 & -0.7 & (j85. & 8 & 0.00 \\
\hline \multirow{6}{*}{1909} & Nor. & 9 & $21 \%$ & 44 & 2.0 & 4 & +0.4 & . itifo & 4 & 0.03 \\
\hline & Jan. & 21 & 3 & 32 & 1.3 & 4 & -0.3 & .666 & 8 & -0.02 \\
\hline & & 22 & $21 / 2$ & 42 & 1.4 & 4 & 0.2 & .720 & 4 & +0.03 \\
\hline & Mar. & 17 & $2 \%$ & 37 & 1.5 & 4 & -0.1 & .753 & 4 & +0.06 \\
\hline & Oct. & 1 & $31 / 2$ & 51 & 2.8 & 4 & +1.2 & 682 & 4 & -0.01 \\
\hline & & 10 & $\begin{array}{l}21 / 2 \\
21 \%\end{array}$ & $\begin{array}{l}56 \\
42\end{array}$ & $\begin{array}{r}0.5 \\
321.1\end{array}$ & 4 & $\begin{array}{l}-1.1 \\
-0.5\end{array}$ & $\begin{array}{r}.647 \\
1.724\end{array}$ & $\begin{array}{l}3 \\
4\end{array}$ & $\begin{array}{l}-0.04 \\
+0.03\end{array}$ \\
\hline \multirow{2}{*}{\multicolumn{3}{|c|}{ Mean_- }} & & \multirow{2}{*}{$48^{\circ}$} & 321.50 & \multirow{2}{*}{\multicolumn{2}{|c|}{ \pm 0.14}} & 1.689 & \multirow{2}{*}{\multicolumn{2}{|c|}{ \pm 0.007}} \\
\hline & & & & & & & & & & \\
\hline
\end{tabular}

618

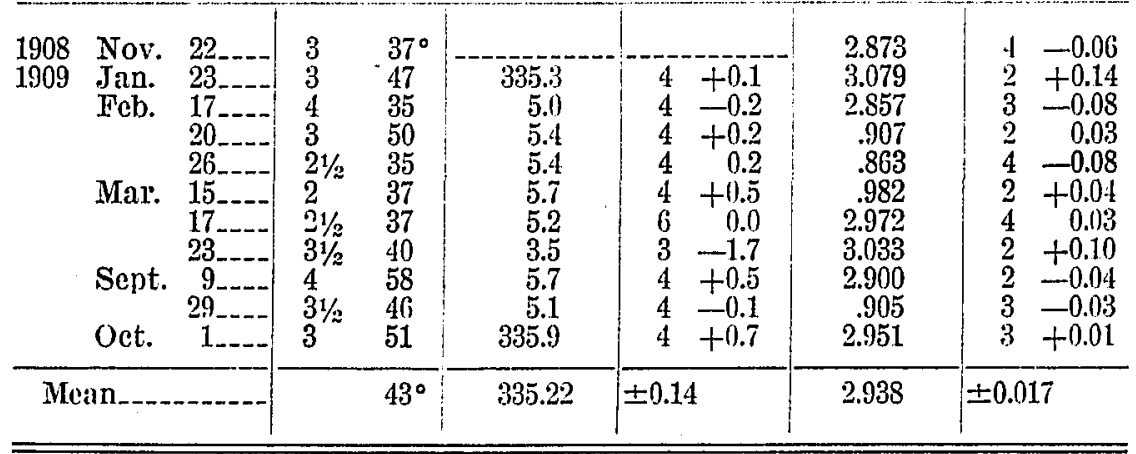


Observations with reference to 628 -Continued.

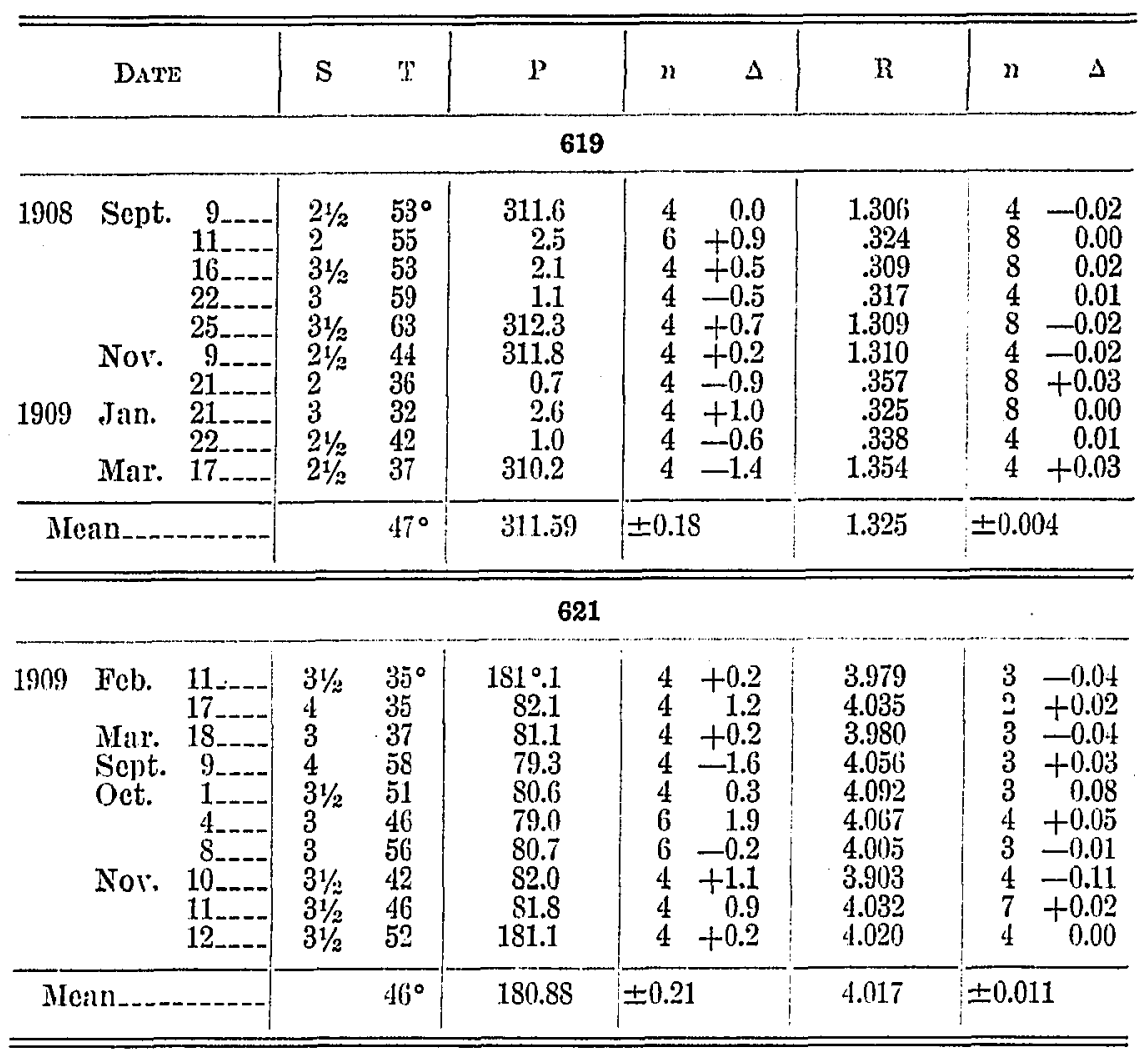

622

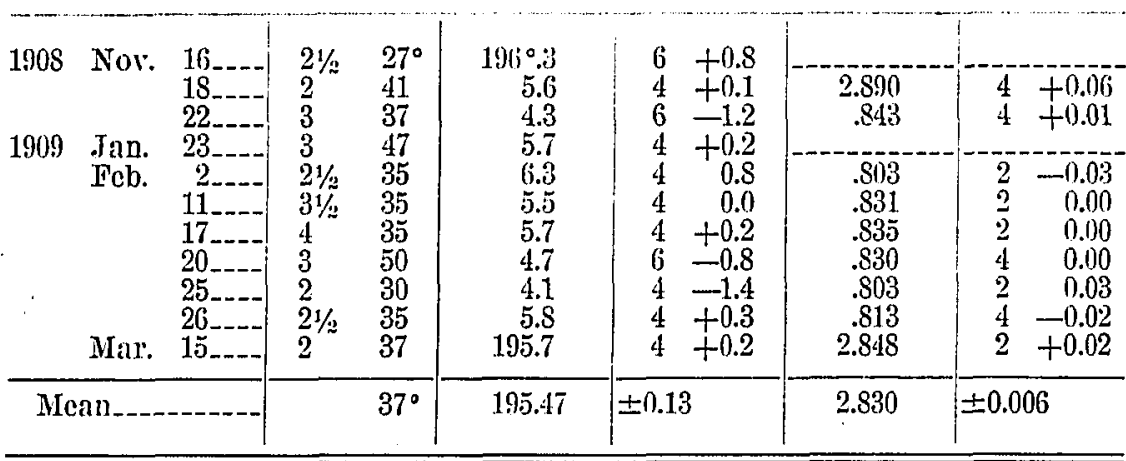


Observations with refercnce to 628-Continued.

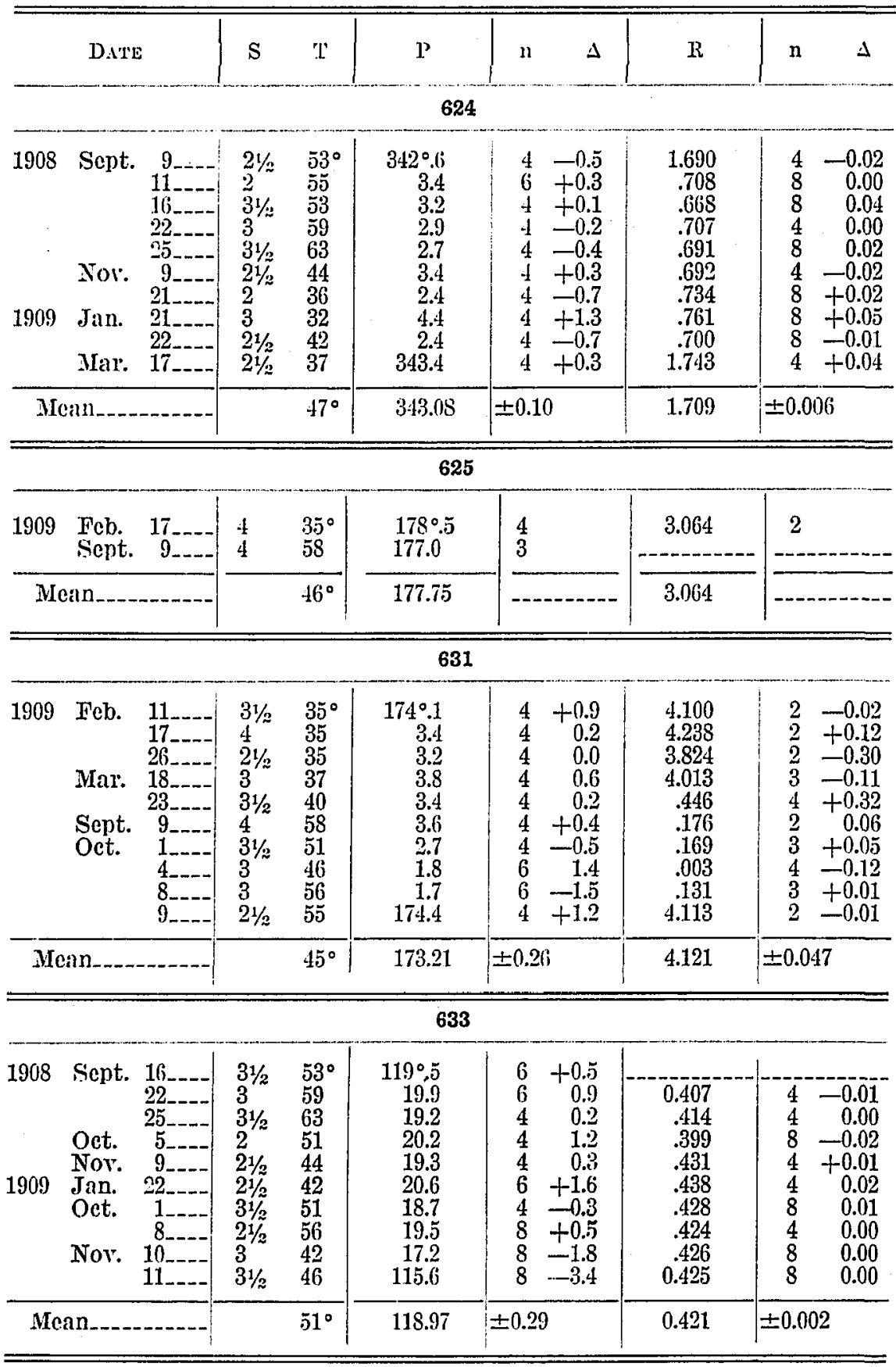


Observations with reference to 6:S-Continued.

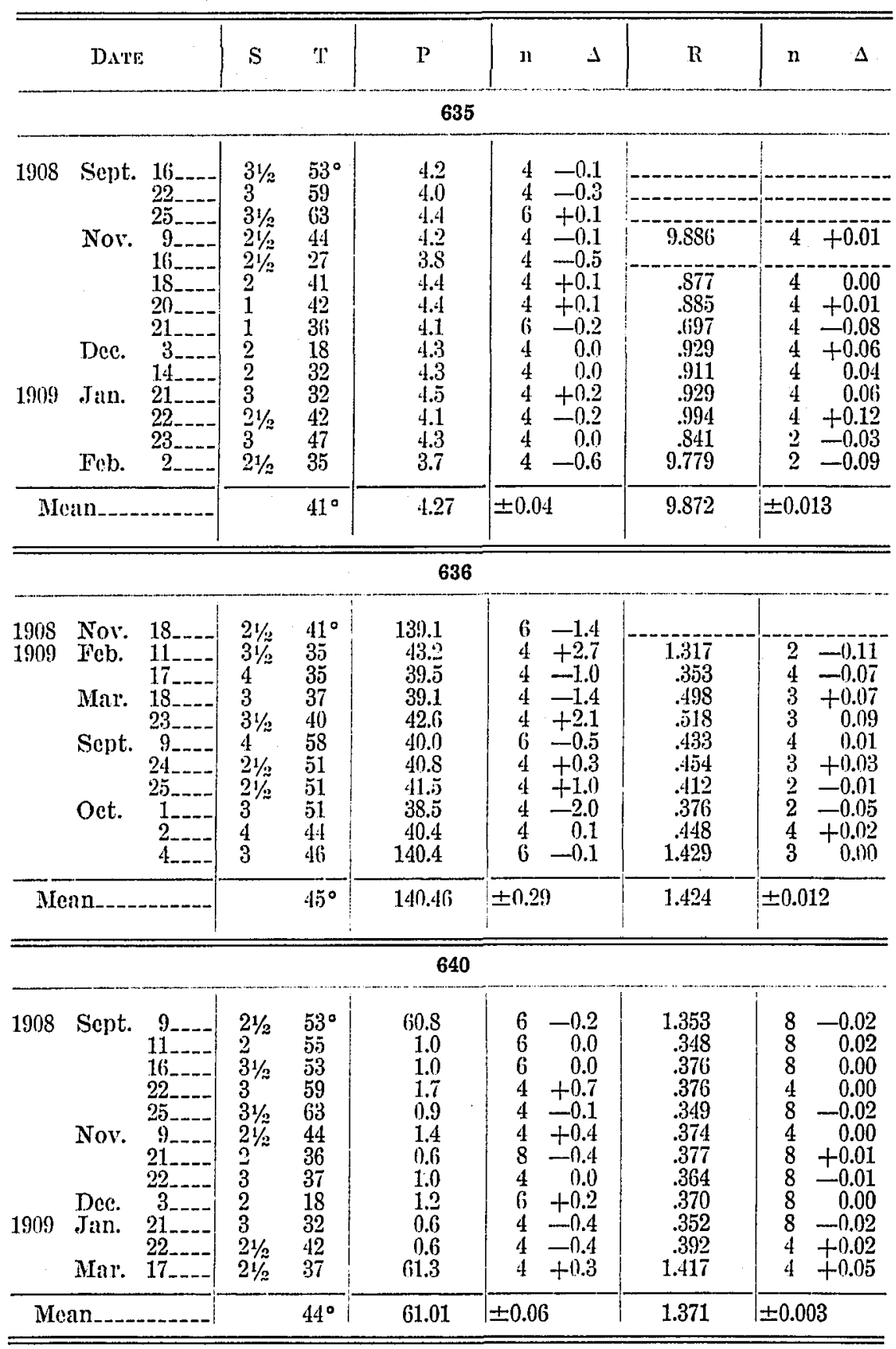


Observations with reference to 628-Continued.

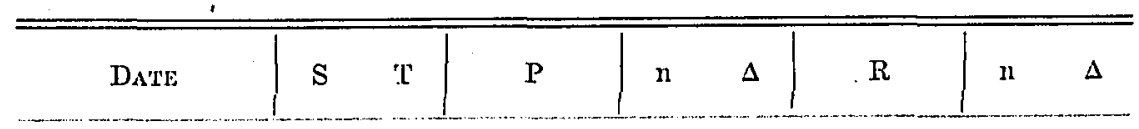

642

\begin{tabular}{|c|c|c|c|c|c|c|c|c|}
\hline 1909 & $\begin{array}{l}\text { Feb. } \\
\text { Sent. } \\
\text { Oet. } \\
\text { Nov. } \\
\text { Feb. }\end{array}$ & $\begin{array}{r}11 \\
14 \\
7 \\
8 \\
10 \\
11 \\
12 \\
1 \ldots\end{array}$ & $\begin{array}{l}31 / 2 \\
4 \\
4 \\
31 / 2 \\
3 \\
31 / 2 \\
31 / 2 \\
31 / 2\end{array}$ & $\begin{array}{l}35^{\circ} \\
60 \\
56 \\
56 \\
42 \\
46 \\
52 \\
33\end{array}$ & $\begin{array}{r}16.4 \\
7.8 \\
5.9 \\
6.0 \\
5.7 \\
5.8 \\
5.6 \\
16.6\end{array}$ & $\begin{array}{rr}4 & +0.2 \\
4 & +1.6 \\
2 & -0.3 \\
0 & 0.2 \\
4 & 0.5 \\
4 & 0.4 \\
4 & -0.6 \\
4 & +0.4\end{array}$ & $\begin{array}{l}4.875 \\
4.853 \\
5.010 \\
5.038 \\
5.021 \\
5.041 \\
4.937 \\
5.009\end{array}$ & $\begin{array}{rr}2 & -0.10 \\
2 & -0.12 \\
2 & +0.04 \\
3 & 0.06 \\
3 & 0.05 \\
4 & +0.07 \\
4 & -0.04 \\
4 & +0.03\end{array}$ \\
\hline & in & _-__ & & $48^{\circ}$ & 16.22 & \pm 0.16 & 4.974 & \pm 0.020 \\
\hline
\end{tabular}

647

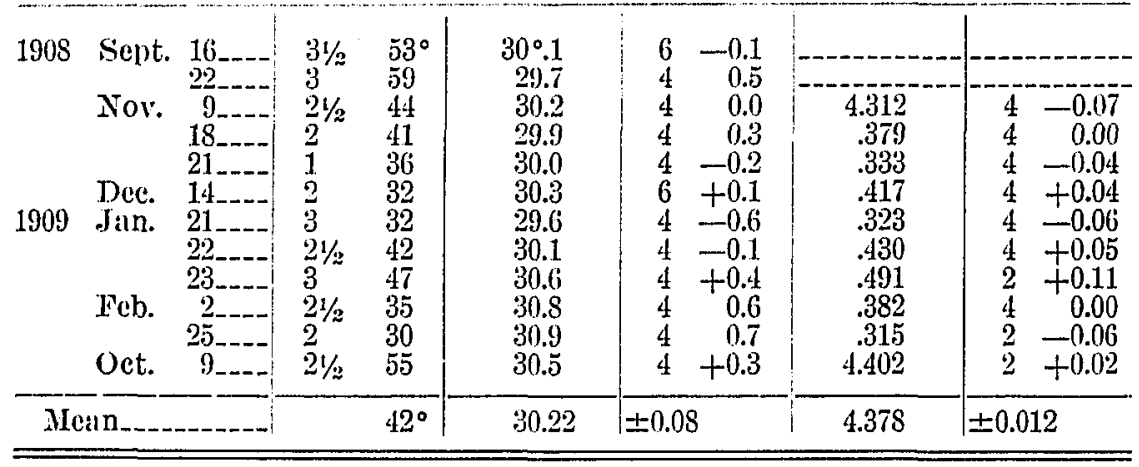

648

\begin{tabular}{|c|c|c|c|c|c|c|c|c|}
\hline 1909 & $\begin{array}{lr}\text { Feb. } & 11 \\
& 17 \\
\text { Mar. } & 23 \\
\text { Sent. } & 9- \\
& 24 \\
& 25 \\
\text { Oct. } & 1 \\
& 2 \\
& 4 \\
& 4 \\
& 8\end{array}$ & $\begin{array}{l}31 / 2 \\
4 \\
31 / 2 \\
4 \\
3 \\
21 / 2 \\
31 / 2 \\
4 \\
3 \\
31 / 2\end{array}$ & $\begin{array}{l}35^{\circ} \\
35 \\
40 \\
58 \\
55 \\
51 \\
51 \\
44 \\
46 \\
56\end{array}$ & $\begin{array}{r}11.5 .9 \\
6.9 \\
6.0 \\
7.8 \\
4.8 \\
4.5 \\
7.8 \\
5.9 \\
5.5 \\
114.9\end{array}$ & $\begin{array}{rr}4 & -0.1 \\
5 & +0.9 \\
4 & 0.0 \\
6 & +1.8 \\
4 & -1.2 \\
4 & -1.5 \\
4 & +1.5 \\
4 & -0.1 \\
4 & 0.5 \\
4 & -1.1\end{array}$ & $\begin{array}{r}2.739 \\
.720 \\
.929 \\
.856 \\
.864 \\
.842 \\
.858 \\
.821 \\
.850 \\
2.868\end{array}$ & $\begin{array}{l}2 \\
2 \\
3 \\
3 \\
4 \\
2 \\
4 \\
3 \\
2 \\
3\end{array}$ & $\begin{array}{r}-0.10 \\
-0.11 \\
+0.09 \\
0.02 \\
0.03 \\
0.01 \\
+0.02 \\
0.01 \\
+0.02 \\
+0.03\end{array}$ \\
\hline \multicolumn{2}{|c|}{ Mean__.......... } & & $47^{\circ}$ & 116.00 & \pm 0.24 & 2.835 & \multicolumn{2}{|c|}{ \pm 0.012} \\
\hline
\end{tabular}


Otservations with reference to 62S-Continued.

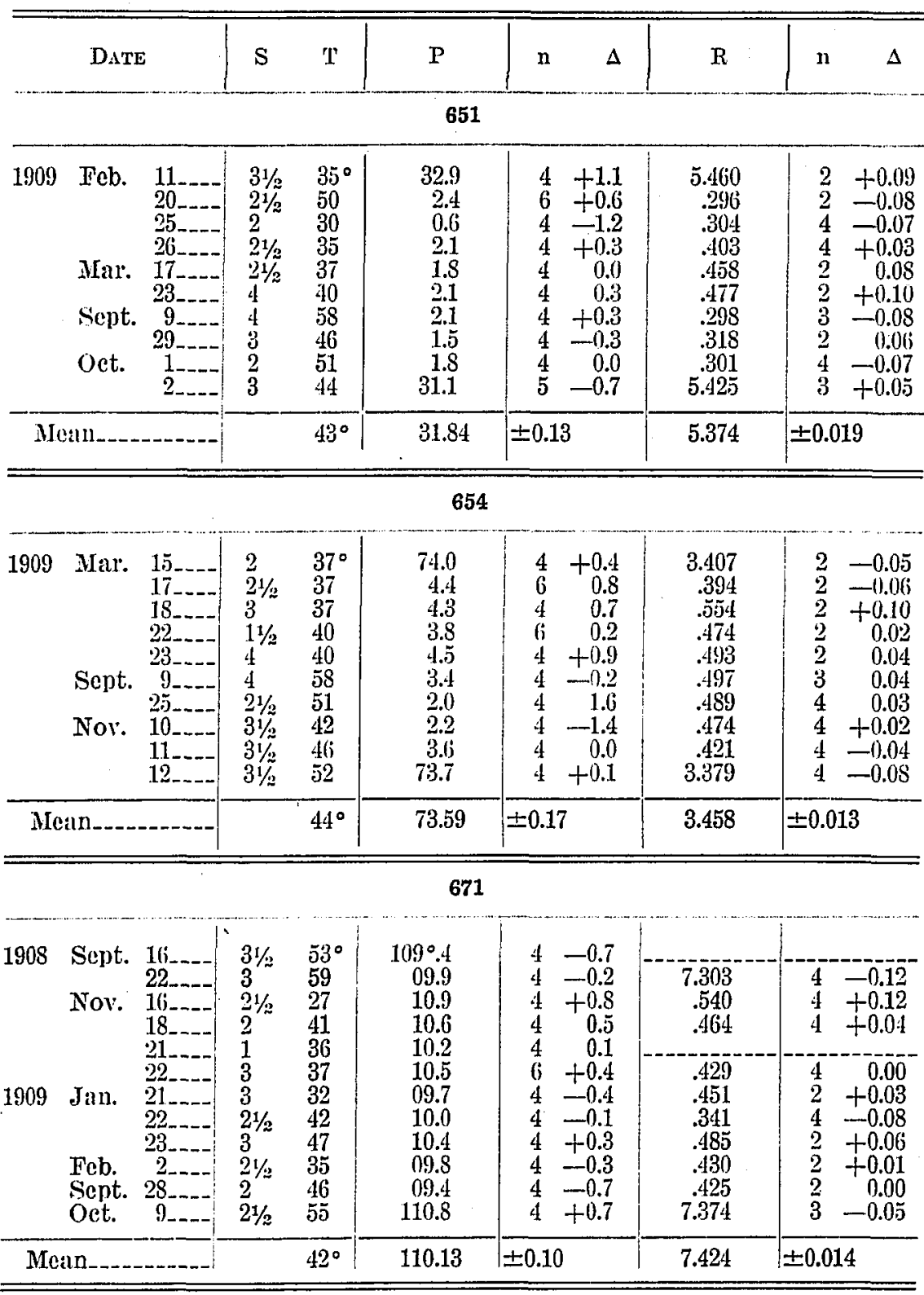


Observalions with reference to 62S-Continued.

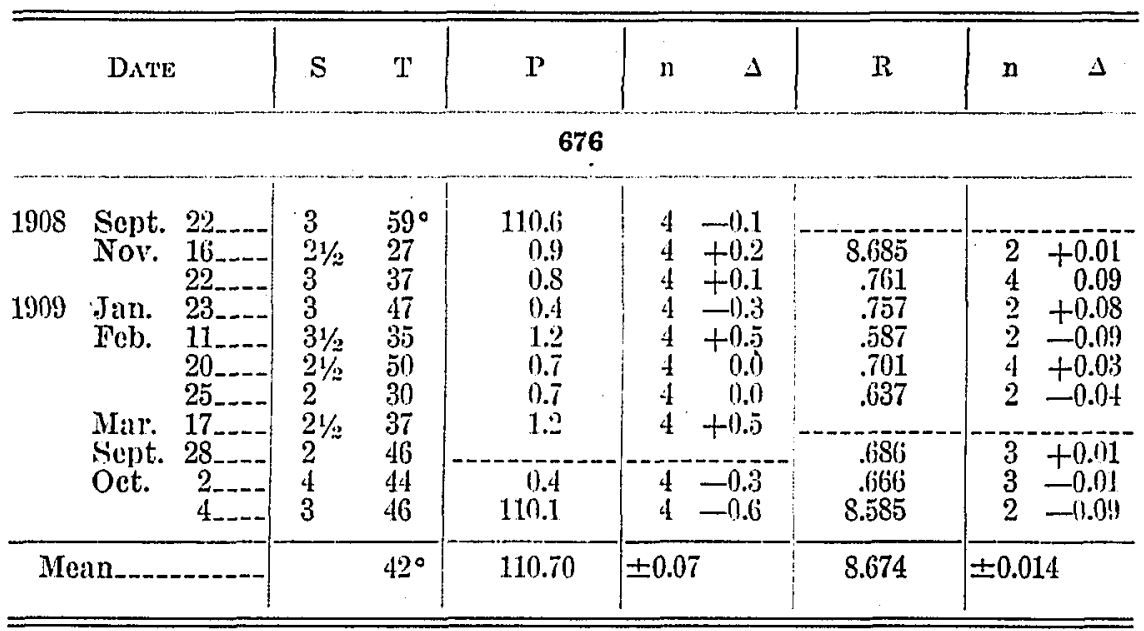

686

(Other mensures referred to 6s5.)

\begin{tabular}{ll|ll|l|l|l|l}
\hline 1909 Feb. $17 .-$ & 4 & $35^{\circ}$ & $125^{\circ} .8$ & 4 & 10.705 & 2 \\
\hline
\end{tabular}

(Other mensures referted to 085.$)$

\begin{tabular}{ll|ll|l|l|l|l}
\hline 1909 Feb. $17 \ldots \ldots$ & 4 & $35^{\circ}$ & $100^{\circ} .1$ & 4 & 13.188 & 3 \\
\hline
\end{tabular}

W 1

\begin{tabular}{|c|c|c|c|c|c|c|c|c|}
\hline 1909 & $\begin{array}{l}\text { Oet. } \\
\text { Nov. }\end{array}$ & $\begin{array}{c}8 \\
11 \\
12 \\
12\end{array}$ & $\begin{array}{l}31 / 2 \\
31 / 2 \\
31 / 2\end{array}$ & $\begin{array}{l}56^{\circ} \\
46^{\circ} \\
52\end{array}$ & $\begin{array}{r}313^{\circ} .4 \\
4.2 \\
4.2\end{array}$ & $\begin{array}{ll}4 & -0.2 \\
4 & +0.1 \\
4 & +0.1\end{array}$ & $\begin{array}{r}12.5666 \\
.704 \\
12.634\end{array}$ & $\begin{array}{rr}2 & -0.07 \\
4 & +0.07 \\
4 & 0.00\end{array}$ \\
\hline 1910 & Feb. & $1 \ldots$ & $31 / 2$ & 33 & 314.0 & $4-0.1$ & & $\cdots$ \\
\hline \multicolumn{3}{|c|}{ Mean } & & $47^{\circ}$ & 314.08 & \pm 0.06 & 12.635 & \\
\hline
\end{tabular}

W 2

\begin{tabular}{|c|c|c|c|c|c|c|c|c|}
\hline 1910 & Nov. & $\begin{array}{r}10 \ldots \\
11 \\
12 \\
1\end{array} \ldots$ & $\begin{array}{l}31 / 2 \\
31 \% 2 \\
31 / 2 \\
31 / 2\end{array}$ & $\begin{array}{l}42^{\circ} \\
46 \\
52 \\
33\end{array}$ & $\begin{array}{r}248.5 \\
9.4 \\
8.0 \\
248.5\end{array}$ & $\begin{array}{ll}4 & -0.1 \\
4 & +0.8 \\
4 & -0.6 \\
4 & -0.1\end{array}$ & $\begin{array}{r}6.832 \\
.728 \\
.869 \\
6.838\end{array}$ & $\begin{array}{l}4+0.02 \\
4+0.09 \\
4+0.05 \\
4+0.02\end{array}$ \\
\hline $\mathrm{Mc}$ & $a n$ & & & $43^{\circ}$ & 248.60 & \pm 0.20 & 6.817 & \pm 0.021 \\
\hline
\end{tabular}


Observalions with reference to 628-Continued.

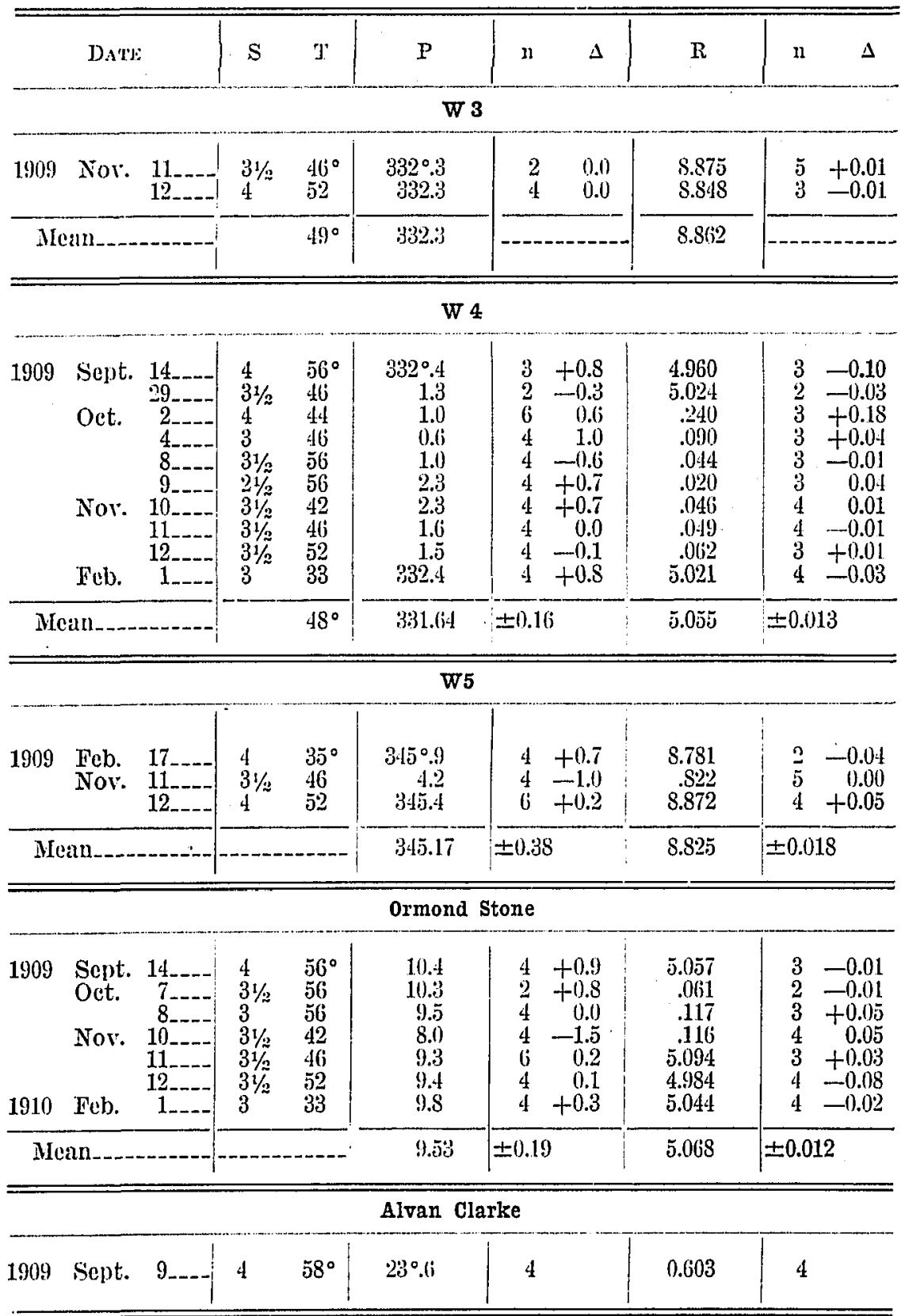


Obscrvations with referenec to $55 \mathrm{~s}$.

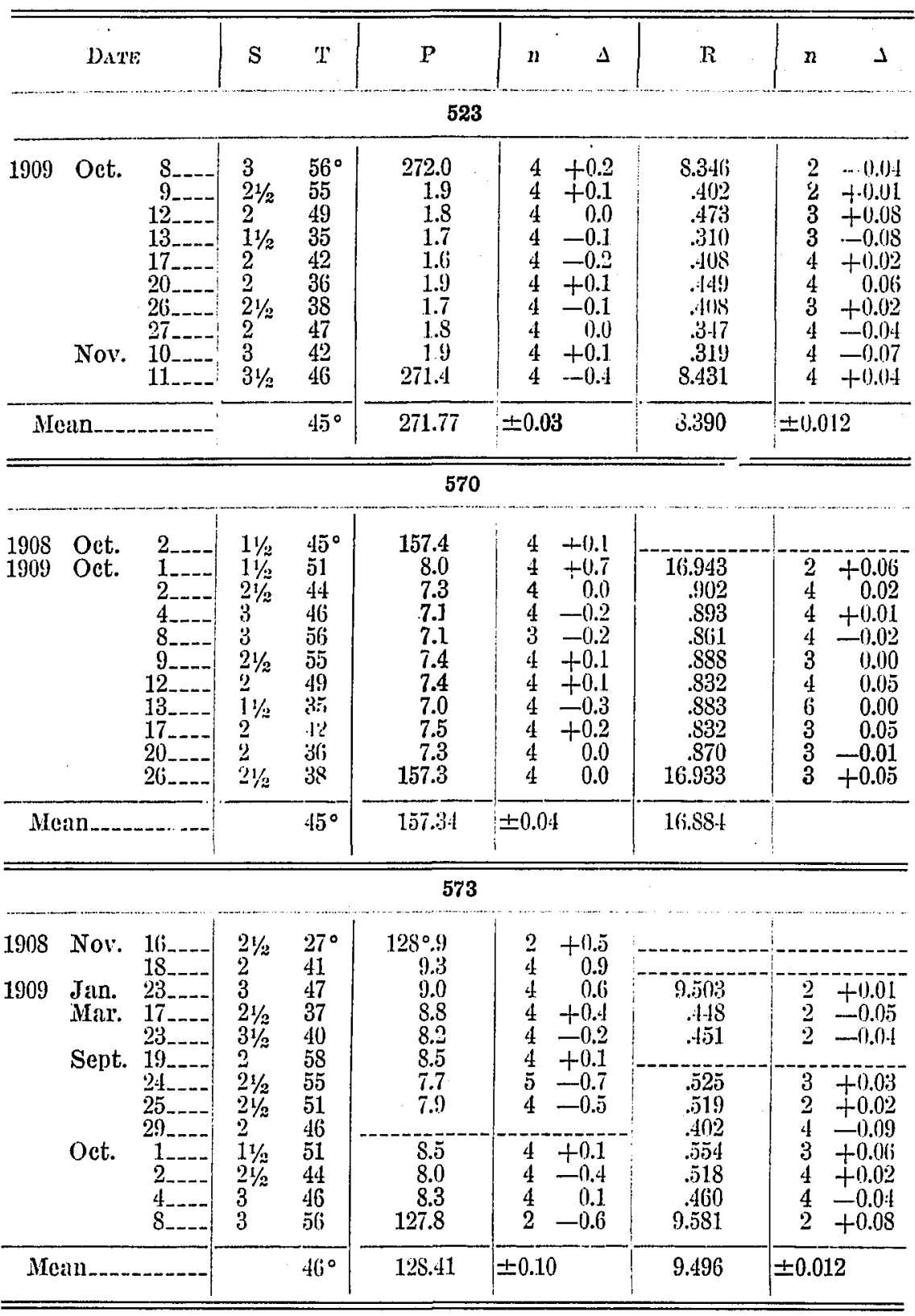


Obscrvations with reference to 558-Continued.

\begin{tabular}{|c|c|c|c|c|c|c|c|c|c|c|}
\hline \multicolumn{3}{|c|}{ Date } & $S$ & $\mathrm{I}$ & $P$ & $\mathrm{n}$ & $\Delta$ & $\mathrm{R}$ & $\mathrm{n}$ & $\Delta$ \\
\hline \multicolumn{11}{|c|}{$\begin{array}{l}\quad 575 \\
\text { teasures referred to }(j 2 \mathrm{~s} \text {.) }\end{array}$} \\
\hline 1908 & Nor. & $16 \ldots$ & $21 / 2$ & 27 & 38.8 & 4 & & 12.264 & 2 & \\
\hline \multicolumn{11}{|c|}{581} \\
\hline 1909 & $\begin{array}{l}\text { Mlar. } \\
\text { Sept. } \\
\text { Oct. }\end{array}$ & $\begin{array}{r}23 \ldots \\
19 \\
24 \\
25 \\
29 \\
29 \\
1 \\
2 \\
4 \\
4 \\
9 \\
9\end{array}$ & $\begin{array}{l}31 / 2 \\
2 \\
21 / 2 \\
21 / 2 \\
2 \\
2 \\
21 / 2 \\
3 \\
3 \\
21 / 2\end{array}$ & $\begin{array}{l}40^{\circ} \\
58 \\
55 \\
51 \\
46 \\
51 \\
44 \\
46 \\
56 \\
55\end{array}$ & $\begin{array}{r}115.3 \\
5.4 \\
5.4 \\
4.9 \\
4.6 \\
4.3 \\
5.5 \\
5.6 \\
5.1 \\
115.5\end{array}$ & $\begin{array}{l}4 \\
4 \\
4 \\
4 \\
4 \\
4 \\
4 \\
4 \\
4 \\
4\end{array}$ & $\begin{array}{r}+0.1 \\
0.2 \\
+0.2 \\
-0.3 \\
0.6 \\
-0.9 \\
+0.3 \\
+0.4 \\
-0.1 \\
+0.3\end{array}$ & $\begin{array}{r}9.878 \\
.746 \\
.884 \\
.871 \\
.6660 \\
9.908 \\
10.1006 \\
9.886 \\
.891 \\
9.859\end{array}$ & $\begin{array}{l}3 \\
2 \\
2 \\
3 \\
3 \\
4 \\
3 \\
4 \\
4 \\
2 \\
3\end{array}$ & $\begin{array}{r}+0.02 \\
+0.11 \\
+0.02 \\
+0.01 \\
+0.20 \\
+0.01 \\
0.14 \\
0.03 \\
+0.03 \\
0.00\end{array}$ \\
\hline $\mathrm{Mc}$ & in & $\cdots$ & & $50^{\circ}$ & 115.16 & \pm 0.0 & & 9.859 & \pm 0.0 & \\
\hline
\end{tabular}

Observalions with reference to 669 .

635

(Other measures referred to lizs.)

\begin{tabular}{|c|c|c|c|c|c|c|c|c|c|}
\hline $\begin{array}{l}1908 \\
1909\end{array}$ & $\begin{array}{l}\text { Nov. } \\
\text { Jan. } \\
\text { Fob. }\end{array}$ & $\begin{array}{r}18 \\
23 \\
4 \\
6 \ldots \\
60 \\
20 \ldots \\
25 \\
26 \ldots \\
\ldots\end{array}$ & $\begin{array}{l}2 \\
3 \\
2 \\
2 \\
21 / 2 \\
2 \\
21 / 2\end{array}$ & $\begin{array}{l}41^{\circ} \\
47 \\
40 \\
35 \\
50 \\
30 \\
35\end{array}$ & $\begin{array}{r}268.8 \\
8.4 \\
9.1 \\
8.4 \\
8.4 \\
5.7 \\
267.6\end{array}$ & $\begin{array}{ll}4 & - \\
4 & - \\
4 & - \\
5 & - \\
4 & - \\
4 & - \\
4 & -\end{array}$ & $\begin{array}{l}+0.3 \\
-0.1 \\
+0.5 \\
-0.1 \\
-0.1 \\
+0.2 \\
-0.9\end{array}$ & $\begin{array}{r}5.627 \\
.655 \\
.743 \\
.681 \\
.595 \\
5.632\end{array}$ & $\begin{array}{rr}4 & -0.03 \\
2 & 0.00 \\
2 & +0.09 \\
4 & +0.02 \\
2 & -0.06 \\
3 & -0.02\end{array}$ \\
\hline \multicolumn{3}{|c|}{ Mean } & & $40^{\circ}$ & $2(6 \mathrm{~S} .47$ & \multicolumn{2}{|l|}{ \pm 0.07} & 5.656 & \pm 0.013 \\
\hline
\end{tabular}

641

\begin{tabular}{|c|c|c|c|c|c|c|c|c|c|}
\hline 1909 & $\begin{array}{l}\text { Jin. } \\
\text { Feb. } \\
\text { Mar. } \\
\text { Sept. }\end{array}$ & 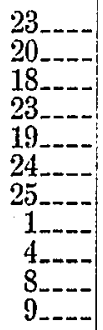 & $\begin{array}{l}3 \\
21 / 2 \\
3 \\
31 / 2 \\
21 / 2 \\
21 / 2 \\
21 / 2 \\
21 / 2 \\
3 \\
3 \\
21 / 2\end{array}$ & $\begin{array}{l}47^{\circ} \\
50 \\
37 \\
40 \\
58 \\
55 \\
51 \\
51 \\
46 \\
56 \\
55\end{array}$ & $\begin{array}{r}282.0 \\
3.9 \\
3.1 \\
2.9 \\
3.5 \\
2.5 \\
2.0 \\
3.5 \\
3.4 \\
3.6 \\
283.6\end{array}$ & $\begin{array}{l}4 \\
4 \\
4 \\
4 \\
4 \\
4 \\
4 \\
4 \\
4 \\
4 \\
4\end{array}$ & $\begin{array}{r}-1.1 \\
+0.8 \\
0.0 \\
-0.2 \\
+0.4 \\
-0.6 \\
-1.1 \\
+0.4 \\
0.3 \\
0.5 \\
+0.5\end{array}$ & $\begin{array}{r}5.4165 \\
.587 \\
.458 \\
.414 \\
.350 \\
.479 \\
.488 \\
.521 \\
.408 \\
5.426\end{array}$ & $\begin{array}{rr}2 & 0.00 \\
2 & +0.12 \\
4 & -0.01 \\
4 & 0.02 \\
2 & -0.11 \\
3 & +0.02 \\
4 & 0.03 \\
3 & +0.06 \\
3 . & -0.05 \\
3 & -0.04\end{array}$ \\
\hline \multicolumn{3}{|c|}{ Mean_-_-_-_-_. } & \multicolumn{2}{|r|}{$48^{\circ}$} & 283.09 & \multicolumn{2}{|c|}{ \pm 0.14} & 5.462 & \pm 0.012 \\
\hline
\end{tabular}


Observations with reference to 669-Continued.

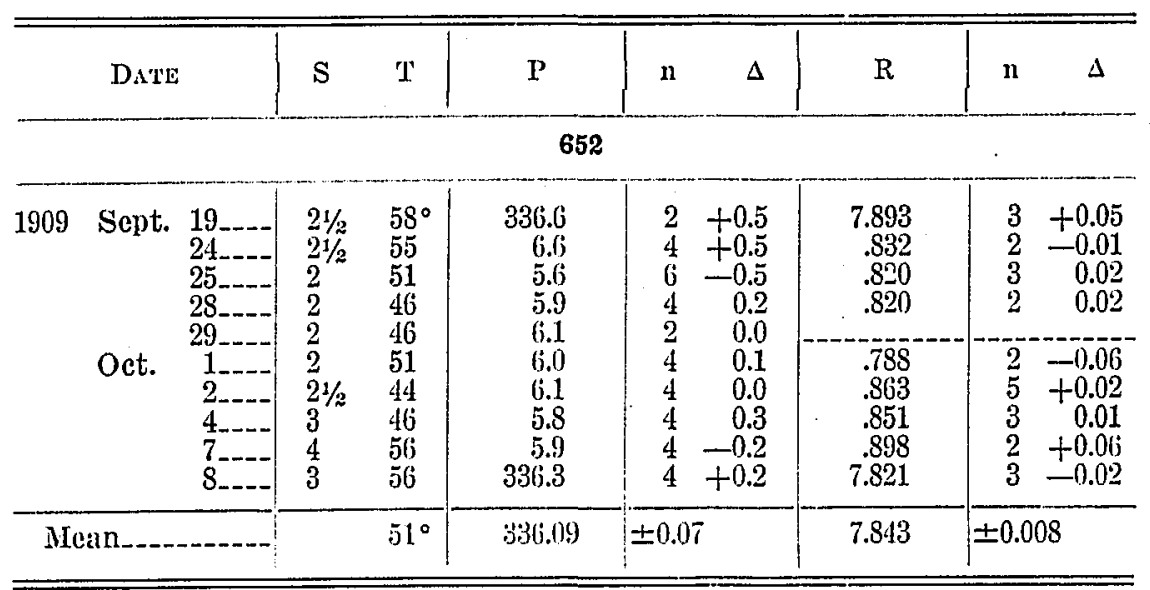

657

\begin{tabular}{|c|c|c|c|c|c|c|c|c|}
\hline 1909 & Sept. & \begin{tabular}{r|}
19 \\
24 \\
25 \\
28 \\
29 \\
1 \\
2 \\
2 \\
$4-$ \\
7 \\
8 \\
8
\end{tabular} & $\begin{array}{l}21 / 2 \\
21 / 2 \\
2 \\
2 \\
2 \\
2 \\
21 / 2 \\
3 \\
4 \\
3\end{array}$ & $\begin{array}{l}58^{\circ} \\
55 \\
51 \\
46 \\
46^{\circ} \\
51 \\
44 \\
46 \\
56 \\
56\end{array}$ & $\begin{array}{r}339.7 \\
40.3 \\
40.5 \\
40.8 \\
40.7 \\
40.3 \\
39.9 \\
39.4 \\
399.3 \\
339.8\end{array}$ & $\begin{array}{rr}2 & -0.4 \\
4 & +0.2 \\
4 & 0.4 \\
4 & 0.7 \\
4 & 0.6 \\
4 & +0.2 \\
4 & -0.2 \\
4 & 0.7 \\
4 & 0.8 \\
4 & -0.3\end{array}$ & $\begin{array}{l}7.012 \\
.072 \\
.049 \\
.158 \\
.076 \\
.086 \\
.066 \\
.054 \\
.120 \\
7.052\end{array}$ & $\begin{array}{lr}2 & -0.06 \\
3 & 0.00 \\
2 & -0.02 \\
3 & +0.08 \\
2 & 0.00 \\
3 & +0.01 \\
4 & -0.01 \\
3 & -0.02 \\
2 & +0.05 \\
2 & -0.02\end{array}$ \\
\hline $\mathrm{Mc}$ & an-.-- & 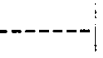 & & $51^{\circ}$ & 340.07 & \pm 0.12 & 7.074 & \pm 0.007 \\
\hline \multicolumn{9}{|c|}{663} \\
\hline 1909 & Sept. & $\begin{array}{r}19 \\
24 \\
25 \\
28 \\
1 \\
2 \\
2 \\
4 \ldots \\
8 \\
9 \\
9 \\
12 . \\
\ldots\end{array}$ & $\begin{array}{l}21 / 2 \\
21 / 2 \\
2 \\
2 \\
21 / 2 \\
21 / 2 \\
3 \\
3 \\
21 / 2 \\
2\end{array}$ & $\begin{array}{l}58^{\circ} \\
55 \\
51 \\
46 \\
51 \\
44 \\
46 \\
56 \\
55 \\
49\end{array}$ & $\begin{array}{r}351.4 \\
0.9 \\
1.7 \\
1.6 \\
1.8 \\
1.8 \\
1.2 \\
0.3 \\
1.2 \\
350.9\end{array}$ & $\begin{array}{rr}4 & +0.1 \\
4 & -0.4 \\
4 & +0.4 \\
4 & 0.3 \\
4 & 0.5 \\
4 & +0.5 \\
4 & -0.1 \\
4 & 1.0 \\
4 & 0.1 \\
4 & -0.4\end{array}$ & $\begin{array}{l}4.827 \\
.826 \\
.840 \\
.880 \\
.758 \\
.867 \\
.843 \\
.832 \\
.813 \\
4.844\end{array}$ & $\begin{array}{ll}3 & -0.01 \\
4 & -0.01 \\
2 & +0.01 \\
3 & +0.05 \\
3 & -0.08 \\
4 & +0.03 \\
3 & +0.01 \\
2 & 0.00 \\
4 & -0.02 \\
3 & +0.01\end{array}$ \\
\hline \multicolumn{3}{|c|}{ Mean } & & $51^{\circ}$ & 351.28 & \pm 0.10 & 4.833 & \pm 0.006 \\
\hline
\end{tabular}


Obscrtations with reference to 669 -Continued.

\begin{tabular}{|c|c|c|c|c|c|c|c|c|c|c|}
\hline & DATE & & $\mathbf{S}$ & $\mathrm{I}$ & $\mathrm{P}$ & $\mathrm{n}$ & $\Delta$ & $\mathrm{R}$ & $\mathbf{n}$ & $\Delta$ \\
\hline \multicolumn{11}{|c|}{681} \\
\hline 1909 & $\begin{array}{l}\text { Sept. } \\
\text { Oet. }\end{array}$ & $\begin{array}{r}19 \\
19 \\
24 \\
25 \\
28 \\
1 \\
2 \\
4 \\
4 \\
8 \\
9 \\
12 \\
12\end{array}$ & $\begin{array}{l}21 / 2 \\
21 / 2 \\
2 \\
2 \\
21 / 2 \\
21 / 2 \\
3 \\
3 \\
21 / 2 \\
2\end{array}$ & $\begin{array}{l}58^{\circ} \\
55 \\
51 \\
46 \\
51 \\
44 \\
46 \\
56 \\
55 \\
49\end{array}$ & $\begin{array}{l}16.8 \\
16.7 \\
17.2 \\
16.3 \\
16.4 \\
16.8 \\
16.6 \\
16.9 \\
16.8 \\
16.5\end{array}$ & $\begin{array}{l}2 \\
4 \\
4 \\
4 \\
4 \\
4 \\
4 \\
4 \\
4 \\
4\end{array}$ & $\begin{array}{r}+0.1 \\
0.0 \\
+0.5 \\
-0.4 \\
-0.3 \\
+0.1 \\
+0.1 \\
+0.2 \\
+0.1 \\
-0.2\end{array}$ & $\begin{array}{l}7.950 \\
8.107 \\
8.076 \\
7.960 \\
8.036 \\
7.996 \\
8.051 \\
7.991 \\
8.011\end{array}$ & $\begin{array}{l}2 \\
3 \\
3 \\
4 \\
4 \\
4 \\
2 \\
3 \\
3\end{array}$ & $\begin{array}{l}-0.07 \\
+0.09 \\
+0.06 \\
-0.06 \\
+0.02 \\
-0.02 \\
+0.03 \\
-0.03 \\
-0.01\end{array}$ \\
\hline \multicolumn{3}{|c|}{ Mean__._._._. } & & $51^{\circ}$ & 16.70 & \multicolumn{2}{|l|}{ \pm 0.05} & 8.018 & \multicolumn{2}{|c|}{ \pm 0.012} \\
\hline
\end{tabular}

Obscrvations wilh refercnec to 685 .

666

\begin{tabular}{|c|c|c|c|c|c|c|c|c|c|}
\hline 1909 & $\begin{array}{l}\text { Sept. } \\
\text { Oct. }\end{array}$ & $\begin{array}{r}19 \\
24 \\
25 \\
1 \\
9 \\
2 \\
4 \\
8- \\
8 \\
9 \\
17-- \\
26-- \\
--\end{array}$ & $\begin{array}{l}21 / 2 \\
2 \\
21 / 2 \\
2 \\
4 \\
3 \\
3 \\
21 / 2 \\
21 / 2 \\
21 / 2\end{array}$ & $\begin{array}{l}58^{\circ} \\
55 \\
51 \\
51 \\
44 \\
46 \\
56 \\
55 \\
42 \\
38\end{array}$ & $\begin{array}{r}201.7 \\
1.9 \\
1.2 \\
0.7 \\
0.9 \\
1.0 \\
1.2 \\
2.1 \\
1.9 \\
201.0\end{array}$ & $\begin{array}{rr}4 & +0.3 \\
5 & +0.5 \\
4 & -0.2 \\
4 & 0.7 \\
4 & 0.5 \\
4 & 0.4 \\
4 & -0.2 \\
4 & +0.7 \\
4 & +0.5 \\
4 & -0.4\end{array}$ & $\begin{array}{l}10.985 \\
11.063 \\
11.020 \\
10.940 \\
10.941 \\
11.027 \\
11.013 \\
11.017 \\
11.016 \\
11.086\end{array}$ & $\begin{array}{l}2 \\
3 \\
2 \\
3 \\
3 \\
4 \\
2 \\
3 \\
3 \\
3\end{array}$ & $\begin{array}{r}-0.03 \\
+0.05 \\
+0.01 \\
-0.07 \\
-0.07 \\
+0.02 \\
0.00 \\
0.01 \\
0.00 \\
+0.08\end{array}$ \\
\hline \multicolumn{3}{|c|}{ Mean } & & $49^{\circ}$ & 201.36 & \pm 0.12 & 11.011 & \multicolumn{2}{|c|}{ \pm 0.011} \\
\hline
\end{tabular}

675

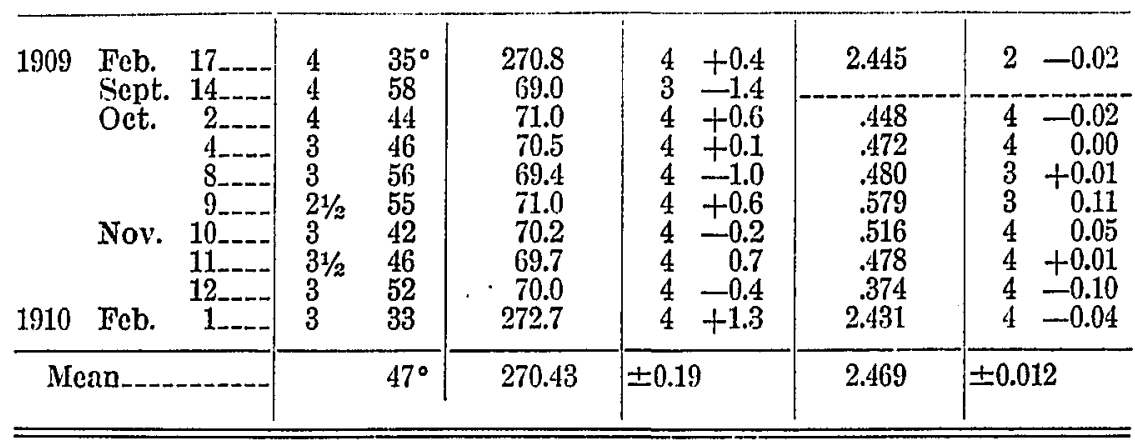


Observations with refcrence to 685 -Continued.

\begin{tabular}{|c|c|c|c|c|c|c|c|c|c|}
\hline & DA'TE & & $\mathrm{S}$ & $\mathrm{T}$ & $\mathrm{P}$ & $\mathrm{n}$ & $\Delta$ & $\mathbf{R}$ & $\Delta$ \\
\hline \multicolumn{10}{|c|}{677} \\
\hline 1909 & $\begin{array}{l}\text { Sept. } \\
\text { Oct. }\end{array}$ & 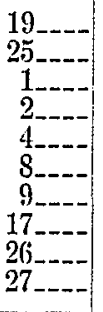 & $\begin{array}{l}21 / 2 \\
21 / 2 \\
2 \\
4 \\
3 \\
3 \\
21 / 2 \\
21 / 2 \\
21 / 2 \\
2^{1 / 2}\end{array}$ & $\begin{array}{l}58^{\circ} \\
51 \\
51 \\
44 \\
46 \\
56 \\
55 \\
42 \\
38 \\
47\end{array}$ & $\begin{array}{r}190.7 \\
89.9 \\
90.4 \\
91.3 \\
90.9 \\
191.6 \\
191.9 \\
1.2 \\
1.2 \\
190.7\end{array}$ & $\begin{array}{l}2 \\
2 \\
4 \\
4 \\
4 \\
4 \\
4 \\
4 \\
4 \\
4 \\
4\end{array}-$ & $\begin{array}{r}-0.3 \\
1.1 \\
-0.6 \\
+0.3 \\
+0.1 \\
+0.6 \\
+0.9 \\
0.2 \\
+0.2 \\
-0.3\end{array}$ & $\begin{array}{r}11.002 \\
10.916 \\
.962 \\
.789 \\
.948 \\
10.943 \\
10.949 \\
10.932 \\
11.080 \\
10.914\end{array}$ & $\begin{array}{rr}4 & +0.06 \\
3 & -0.03 \\
3 & +0.02 \\
3 & -0.16 \\
4 & 0.00 \\
2 & 0.00 \\
3 & 0.00 \\
4 & -0.01 \\
4 & +0.14 \\
3 & -0.03\end{array}$ \\
\hline \multicolumn{3}{|c|}{ Mean_......... } & & $49^{\circ}$ & 190.98 & \pm 0.12 & & 10.944 & \pm 0.012 \\
\hline \multicolumn{10}{|c|}{686} \\
\hline 1910 & $\begin{array}{l}\text { Feb. } \\
\text { Scpt. } \\
\text { Oct. } \\
\text { Nov. } \\
\text { Feb. }\end{array}$ & 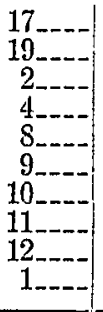 & $\begin{array}{l}4 \\
21 / 2 \\
4 \\
3 \\
31 / 2 \\
21 / 2 \\
31 / 2 \\
31 / 2 \\
3 \\
3\end{array}$ & $\begin{array}{l}35^{\circ} \\
58 \\
44 \\
46 \\
56 \\
55 \\
49 \\
46 \\
52 \\
33\end{array}$ & $\begin{array}{r}344.1 \\
4.5 \\
3.5 \\
3.1 \\
3.4 \\
2.4 \\
2.4 \\
3.2 \\
2.8 \\
2.6 \\
342.8\end{array}$ & $\begin{array}{l}4 \\
2 \\
4 \\
4 \\
4 \\
4 \\
4 \\
6 \\
4 \\
4 \\
4\end{array}-$ & $\begin{array}{r}+0.9 \\
1.3 \\
+0.3 \\
+0.1 \\
+0.2 \\
-0.8 \\
0.0 \\
0.4 \\
0.6 \\
-0.4\end{array}$ & $\begin{array}{l}3.440 \\
.456 \\
.754 \\
.694 \\
.670 \\
.524 \\
.563 \\
.549 \\
.470 \\
3.446\end{array}$ & $\begin{array}{rr}2 & -0.12 \\
2 & -0.10 \\
4 & +0.19 \\
4 & 0.13 \\
3 & +0.11 \\
3 & -0.04 \\
3 & +0.01 \\
4 & -0.01 \\
4 & 0.09 \\
4 & -0.11\end{array}$ \\
\hline \multicolumn{3}{|c|}{ Mean_-_---_-_-- } & & $47^{\circ}$ & 343.24 & \pm 0.14 & & 3.5556 & \pm 0.026 \\
\hline \multicolumn{10}{|c|}{688} \\
\hline 1909 & $\begin{array}{l}\text { Nov. } \\
\text { Feb. }\end{array}$ & $\begin{array}{r}2 \ldots \\
4 \ldots \\
8 \ldots \\
9 \\
11 \\
12 \ldots \\
1 \ldots\end{array}$ & $\begin{array}{l}4 \\
3 \\
31 / 2 \\
3 \\
31 / 2 \\
31 / 2 \\
31 / 2 \\
\end{array}$ & $\begin{array}{l}44^{\circ} \\
46 \\
56 \\
55 \\
46 \\
52 \\
33\end{array}$ & $\begin{array}{r}2.3 .6 \\
4.1 \\
3.4 \\
3.4 \\
3.8 \\
3.1 \\
21.7\end{array}$ & $\begin{array}{l}5 \\
4 \\
4 \\
4 \\
4 \\
4 \\
4\end{array}$ & $\begin{array}{r}-0.1 \\
+0.4 \\
-0.3 \\
-0.3 \\
+0.1 \\
+0.6 \\
+1.0\end{array}$ & $\begin{array}{r}7.913 \\
.957 \\
.993 \\
.979 \\
.936 \\
7.882 \\
8.082\end{array}$ & $\begin{array}{ll}3 & -0.05 \\
4 & -0.01 \\
3 & +0.03 \\
3 & +0.02 \\
4 & -0.03 \\
4 & -0.08 \\
3 & +0.12\end{array}$ \\
\hline $\mathrm{Me}$ & an-..-.-. & ---- & & $48^{\circ}$ & 23.73 & \pm 0.14 & & 7.963 & \pm 0.017 \\
\hline
\end{tabular}


Observations with reference to 685 -Continued.

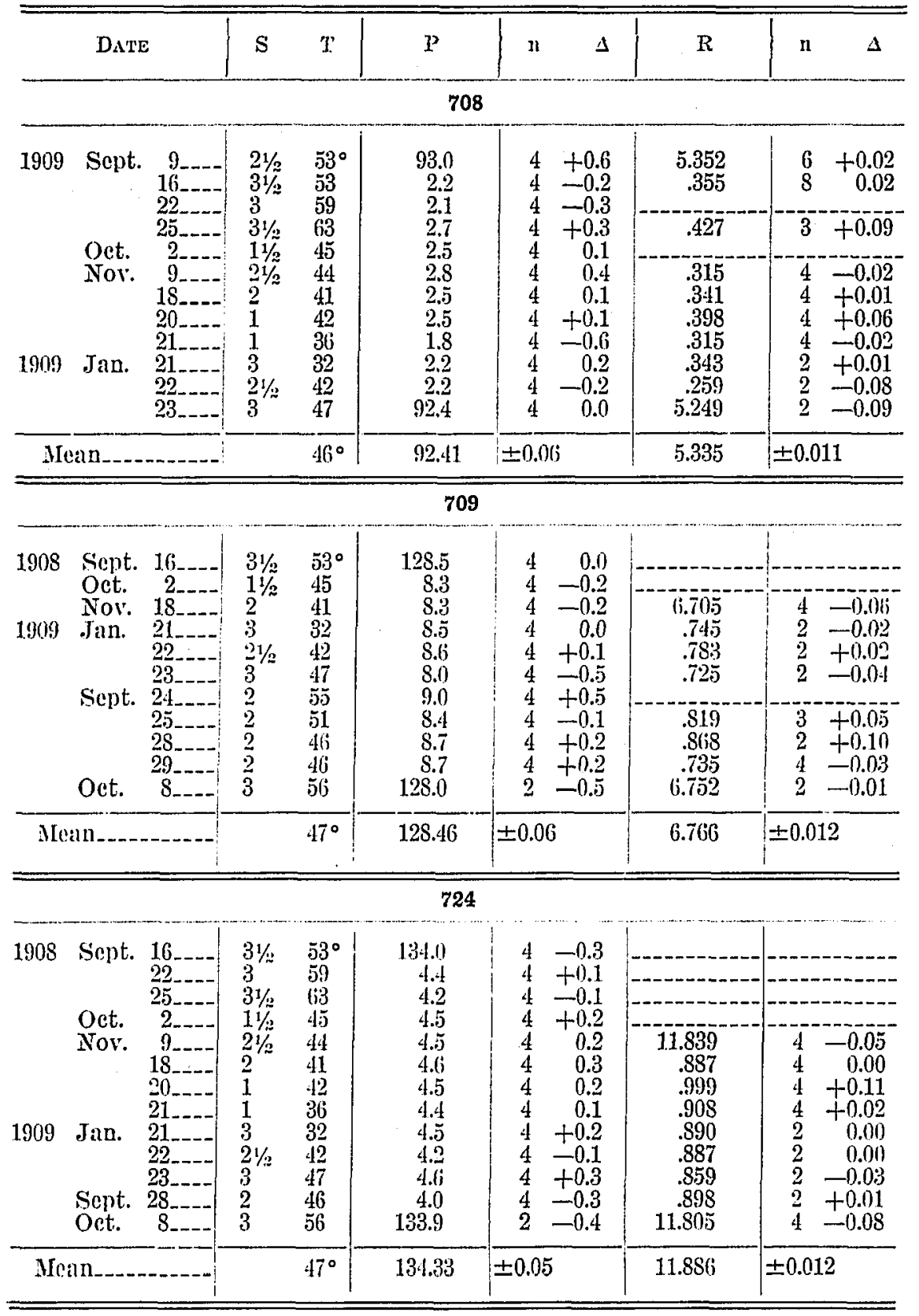


Observations wilh reforence to 685 -Continued.

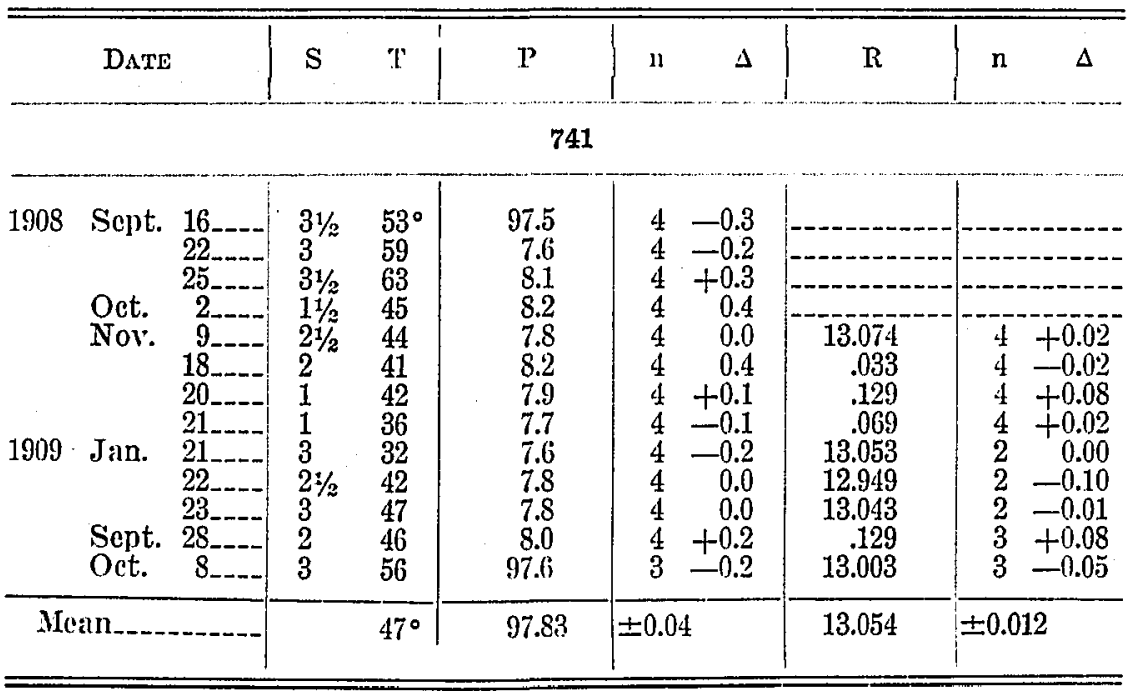

II iscellaneous Observations.

640-619

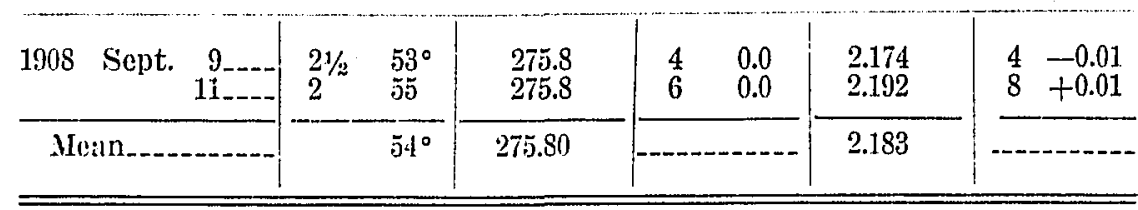

640-624

\begin{tabular}{|c|c|c|c|c|c|c|c|c|c|c|}
\hline 1908 & Sept. & $1{ }^{9}$ & $2^{21 / 2}$ & $\overline{5}^{5 \circ}$ & $\begin{array}{l}300.4 \\
300.5\end{array}$ & $\begin{array}{l}4 \\
4\end{array}$ & $\begin{array}{l}0.0 \\
0.0\end{array}$ & $\begin{array}{l}1.948 \\
1.943\end{array}$ & $\begin{array}{l}4 \\
8\end{array}$ & $\begin{array}{l}0.00 \\
0.00\end{array}$ \\
\hline & an & 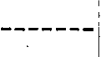 & & $54^{\circ}$ & 300.45 & $-\ldots$ & & 1.946 & --- & \\
\hline
\end{tabular}

640-633

\begin{tabular}{ll|ll|l|l|l|l}
\hline 1908 Sept. 22---- & 3 & $59^{\circ}$ & 225.6 & 4 & 1.195 & 4 \\
\hline
\end{tabular}


Miscellancons Observations-Continued.

\begin{tabular}{|c|c|c|c|c|c|c|c|c|}
\hline & DATE: & $\mathrm{S}$ & 'I' & $\mathrm{P}$ & $\Delta$ & $\mathrm{R}$ & u & $\Delta$ \\
\hline \multicolumn{9}{|c|}{$619-624$} \\
\hline 1908 & Sept. $\begin{array}{l}9 \\
11 \\
25\end{array}$ & $\begin{array}{l}21 / 2 \\
21 \% 2\end{array}$ & $\begin{array}{l}53^{\circ} \\
55 \\
63^{\circ}\end{array}$ & $\begin{array}{l}31.9 \\
33.9 \\
33.0\end{array}$ & $\begin{array}{l}4-1.0 \\
4+1.0 \\
4+0.1\end{array}$ & $\begin{array}{l}0.877 \\
0.892 \\
0.876\end{array}$ & $\begin{array}{l}6 \\
8 \\
8\end{array}$ & $\begin{array}{r}0.001 \\
+0.01 \\
-0.01\end{array}$ \\
\hline \multicolumn{2}{|c|}{ Mean } & & $56^{\circ}$ & 32.93 & - & 0.882 & -- & \\
\hline
\end{tabular}

619-617

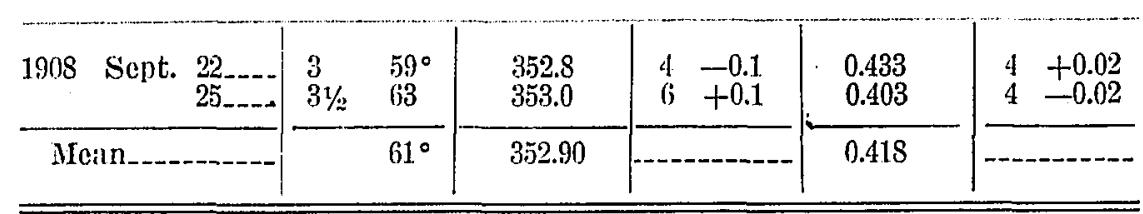

$624-617$

\begin{tabular}{ll|l|l|l|l|l|l|l}
\hline 1908 Sept. 22_.- & 3 & $59^{\circ}$ & 240.1 & 6 & 0.619 & 4 \\
\hline
\end{tabular}

$628-570$

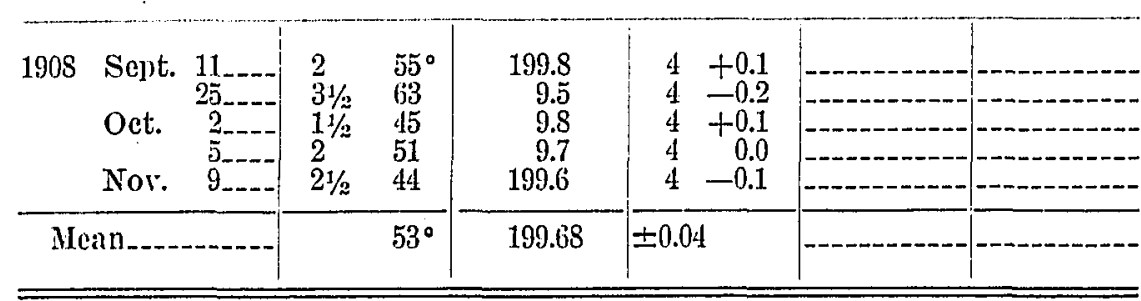


Ifiscellaneous Observations-Continued.

\begin{tabular}{|c|c|c|c|c|c|c|c|c|c|}
\hline & DA'TE & & $s$ & $\mathrm{~T}$ & $P$ & $n$ & $\Delta$ & $\mathrm{R}$ & $\Delta$ \\
\hline \multicolumn{10}{|c|}{$628-708$} \\
\hline 1908 & $\begin{array}{l}\text { Sept. } \\
\text { Nov. }\end{array}$ & 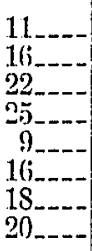 & $\begin{array}{l}2 \\
31 / 2 \\
3 \\
31 / 2 \\
21 / 2 \\
21 / 2 \\
2 \\
1\end{array}$ & $\begin{array}{l}55^{\circ} \\
53 \\
59 \\
63 \\
44 \\
27 \\
41 \\
42\end{array}$ & $\begin{array}{r}123.1 \\
2.4 \\
2.6 \\
2.8 \\
3.1 \\
2.9 \\
3.0 \\
122.5\end{array}$ & $\begin{array}{l}4 \\
4 \\
4 \\
4 \\
4 \\
4 \\
4 \\
4\end{array}$ & $\begin{array}{r}+0.3 \\
-0.1 \\
-0.2 \\
0.0 \\
+0.3 \\
0.1 \\
+0.2 \\
-0.3\end{array}$ & $\begin{array}{r}-18.175 \\
\hdashline 18.019\end{array}$ & $\frac{8+0.08}{-4-0.08}$ \\
\hline \multicolumn{3}{|c|}{ Meann............. } & & $48^{\circ}$ & 122.80 & \pm 0.07 & & 18.097 & \pm 0.048 \\
\hline
\end{tabular}

$628-724$

\begin{tabular}{|c|c|c|c|c|c|c|c|}
\hline Sept. & $\begin{array}{r}11 \\
16 \\
22 \\
25 \\
9 \\
18 \\
19\end{array}$ & $\begin{array}{l}2 \\
31 / 2 \\
3 \\
31 / 2 \\
21 / 2 \\
2\end{array}$ & $\begin{array}{l}55^{\circ} \\
53 \\
59 \\
63 \\
44 \\
41\end{array}$ & $\begin{array}{r}134.5 \\
4.1 \\
3.9 \\
4.3 \\
4.4 \\
134.4\end{array}$ & $\begin{array}{lr}4 & +0.2 \\
4 & -0.2 \\
4 & -0.4 \\
4 & 0.0 \\
4 & +0.1 \\
4 & +0.1\end{array}$ & 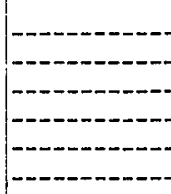 & 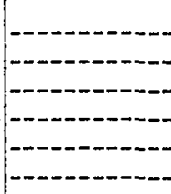 \\
\hline Moan. & & & $52^{\circ}$ & 134.27 & \pm 0.06 & & $\ldots$ \\
\hline
\end{tabular}

628-741

\begin{tabular}{|c|c|c|c|c|c|c|c|c|c|}
\hline 1908 & $\begin{array}{l}\text { Sept. } \\
\text { Nov. }\end{array}$ & $\begin{array}{r}11 \\
16 \\
22 \\
25 \\
9 \\
9 \\
18 \\
-\ldots-\end{array}$ & $\begin{array}{l}2 \\
31 / 2 \\
3 \\
31 / 2 \\
51 / 2 \\
2\end{array}$ & $\begin{array}{l}55^{\circ} \\
53 \\
59 \\
63 \\
44 \\
41\end{array}$ & $\begin{array}{r}116.6 \\
6.2 \\
6.2 \\
6.3 \\
6.6 \\
116.6\end{array}$ & $\begin{array}{l}4 \\
4 \\
4 \\
4 \\
4 \\
4\end{array}$ & $\begin{array}{r}+0.2 \\
+0.2 \\
0.2 \\
-0.1 \\
+0.2 \\
+0.2\end{array}$ & - & מי \\
\hline Me & $\pi 0$ & . & & $52^{\circ}$ & 116.42 & \pm 0.06 & & & \\
\hline
\end{tabular}


JEW POSITIONS OT STARS IN ORION

PAR'L IV.

Catalogue of Positions.

\begin{tabular}{|c|c|c|c|c|c|c|c|c|c|c|}
\hline No. & G.P.B. & H.-S. & II. & W.C.13 & IJl. & Li. & Mag. & $\Delta a$ & $\Delta \delta$ & N. \\
\hline 1 & 523 & 45 & $\tau$ & 5 & 40 & 1 & & $-243^{\prime \prime}, 55$ & $-115^{\prime \prime} 32$ & 10 \\
\hline 2 & $55 \mathrm{~S}$ & 50 & $\ldots$ & 9 & 39 & $v$ & 10.7 & 160.91 & 117.87 & 120 \\
\hline$\overline{3}$ & 567 & 51 & - & 10 & & & 13.3 & 99.79 & 4.78 & 12 \\
\hline 4 & 570 & 53 & $\sigma$ & 13 & 33 & n & & 96.80 & 271.42 & 16 \\
\hline 5 & 573 & 54 & $-\cdots$ & 12 & 35 & $\bar{n}_{i}$ & 12.4 & 87.58 & -176.01 & 12 \\
\hline 6 & $W_{5}$ & $\cdots$ & & & & & & 86.63 & +89.46 & 4 \\
\hline 7 & $\begin{array}{l}575 \\
581\end{array}$ & act54 & $-\cdots$ & 11 & 45 & & 12.0 & $\begin{array}{l}85.59 \\
7399\end{array}$ & -22.28 & 11 \\
\hline $\begin{array}{l}8 \\
9\end{array}$ & $W_{n}$ & & & & & & & 62.57 & $\begin{array}{r}24.52 \\
24.0\end{array}$ & $\begin{array}{r}10 \\
4\end{array}$ \\
\hline 10 & $589^{2}$ & 57 & $--\ldots$ & 10 & & & 12.4 & 57.42 & 21.03 & 12 \\
\hline 11 & 595 & - & & .20 & $13, \mathrm{c}$ & & 13.2 & 46.57 & -16.36 & 8 \\
\hline 12 & $\bar{W}_{\mathrm{s}}$ & -.-- & & & & & & 40.59 & +77.31 & 2 \\
\hline 13 & 601 & --- & & $-\infty$ & & & 13.6 & 32.93 & -28.36 & $\overline{7}$ \\
\hline 14 & 602 & -..- & & & & & 13.4 & 32.84 & -61.58 & 10 \\
\hline 15 & $W_{4}$ & ...... & & & & & & 23.66 & +43.82 & 10 \\
\hline 16 & 608 & ------ & & & $\mathbf{t}$ & & 13.1 & 23.18 & -16.22 & 10 \\
\hline 17 & $W_{s}$ & -..--.- & & & & & & 22.26 & +84.08 & 3 \\
\hline 18 & 612 . & - & $\ldots$ & 16 & & & 13.0 & 18.18 & 26.35 & 10 \\
\hline 19 & 618 & & & 19 & h & & 12.8 & 12.14 & 26.39 & 11 \\
\hline 20 . & 617 & 64 & $\gamma^{\prime}$ & & & $\mathrm{bb}_{1}$ & 10.3 & 10.34 & 13.04 & 11 \\
\hline 21 & 619 & 65 & $\gamma$ & 17 & $\ldots$ & $\mathrm{b}$ & 7.4 & 9.76 & $\begin{array}{r}+8.66 \\
\end{array}$ & 10 \\
\hline 22 & 622 & II & -..- & is & & & 12.0 & 7.44 & -26.88 & 11 \\
\hline 23 & 621 & ad III & & & & & 14.0 & 6.08 & -39.59 & 10 \\
\hline 24 & 624 & 67 & $\delta$ & 21 & & d & 5.7 & 4.90 & +16.11 & 10 \\
\hline 25 & 625 & ad II & -- & - & $d$ & & & -1.18 & -30.14 & 2 \\
\hline 26 & 628 & & $a$ & 22 & & a & 5.7 & 1938 & 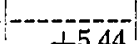 & \\
\hline $\begin{array}{l}27 \\
28\end{array}$ & 633 & 71 & $\overline{a^{\prime}}$ & & & & 10.0 & $\begin{array}{r}+2.00 \\
3.60\end{array}$ & $\begin{array}{r}+2.44 \\
-2.00\end{array}$ & 10 \\
\hline 29 & 631 & & & & & & 13.6 & $4.80^{\circ}$ & -40.32 & 10 \\
\hline 30 & 635 & 70 & . & 23 & 2 & $i$ & 9.5 & 7.20 & +97.40 & 16 \\
\hline 31 & O. Stone & & $-\ldots$ & & & & 14.2 & 8.27 & +49.27 & 7 \\
\hline 32 & 636 & & $\ldots$ & 24 & & & 13.4 & 8.93 & -10.82 & 11 \\
\hline 33 & 64 & III & . & & & & 12.8 & 10.44 & +111.33 & 11 \\
\hline 34 & 640 & 73 & $\theta$ & 25 & & c & 7.1 & 11.82 & 6.55 & 12 \\
\hline 35 & 642 & & & & & & 13.7 & 13.0 & 47.03 & 8 \\
\hline 36 & 647 & 75 & $\ldots$ & $2 i$ & 9,1 & $\cdots$ & 11.0 & 21.72 & +37.29 & 12 \\
\hline 37 & 64 & -1 & & & & $\ldots$ & 13.7 & 25.11 & -12.25 & 10 \\
\hline 38 & 651 & ad 75 & & 27 & & & 12.3 & 27.94 & +44.99 & 10 \\
\hline 39 & 652 & 76 & $\mathrm{y}^{\prime}$ & 32 & & $\overline{\mathrm{I}}=$ & 11.7 & 31.54 & 169.80 & 10 \\
\hline 40 & 65 & 78 & & 31 & & & 13.1 & 32.69 & 9.63 & 10 \\
\hline 41 & 65 & 80 & $y^{\prime \prime}$ & 33 & 4 & $f=$ & 11.3 & 39.10 & 164.68 & ii) \\
\hline 42 & 60 & 84 & $\omega$ & 37 & & $f^{\prime \prime}$ & 12.8 & 55.65 & +146.22 & 10 \\
\hline 43 & 66 & S1. & & 30 & & & 13.4 & 57.43 & -195.10 & 10 \\
\hline 44 & 66 & 87 & $y$ & 39 & 10 & k & 8.8 & 62.87 & $\begin{array}{r}+99.20 \\
\end{array}$ & 120 \\
\hline 45 & 671 & 88 & $\ldots$ & 41 & is & $e^{\prime \prime}$ & 11.2 & 68.69 & -25.18 & 10 \\
\hline 46 & 67 & & & & $\mathbf{a}$ & $n^{\circ}$ & & 72.63 & 92.21 & 10 \\
\hline 47 & 677 & $\operatorname{ad} 81$ & -- & 34 & & 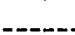 & 13.8 & 76.42 & 199.93 & 10 \\
\hline 48 & 676 & ad 88 & $\%$ & 43 & $\mathrm{k}$ & & 12.8 & 79.96 & -30.22 & 11 \\
\hline 49 & 68 & 89 & & & & e" & & & +174.83 & 10 \\
\hline 50 & 68 & 91. & $\epsilon$ & 44 & & & 13.4 & 95.95 & -60.49 & 11 \\
\hline 51 & 685 & 93 & ...... & 45 & 26 & c & - & 96.96 & 94.04 & 120 \\
\hline 52 & 68 & & & & & & & & & \\
\hline 53 & 709 & 100 & $G$ & 51 & & $\mu$ & 12.0 & 149.17 & 135.52 & 11 \\
\hline 54 & 708 & 101 & $\xi$ & 50 & 23 & $f$ & 6.3 & 149.48 & 96.25 & 20 \\
\hline 5 & 724 & 104 & $\lambda$ & (j) & 25 & $\mathrm{~h}$ & 8. & & 175.92 & 19 \\
\hline 56 & 74 & 110 & $\eta$ & 61 & 19 & $g$ & 7.9 & +224.41 & -111.57 & 19 \\
\hline
\end{tabular}


UNIVIRSITY OF VIRGINIA PUBLICATIONS

PAI'I $V$.

l'ositions of Other Observers Reduced to 1910.0.

\begin{tabular}{|c|c|c|c|c|c|c|c|}
\hline Still & $\Delta a$ & $\Delta \delta$ & Ous. & Star & $\Delta a$ & $\Delta \delta$ & Obs. \\
\hline \multirow[t]{3}{*}{523} & \multirow{2}{*}{$\begin{array}{r}-246.6 \\
244.2 \\
243.5 \\
243.8 \\
-245.8\end{array}$} & \multirow{2}{*}{$\begin{array}{r}-114.6 \\
115.6 \\
116.6 \\
114.8 \\
-115.5\end{array}$} & \multirow{2}{*}{$\begin{array}{c}\mathrm{H} \\
\mathrm{WB} \\
\mathrm{LB} \\
\mathrm{LBS}\end{array}$} & 581 & $\begin{array}{r}-77.6 \\
73.3\end{array}$ & $\begin{array}{r}-158.7 \\
109.9\end{array}$ & $\stackrel{G B}{W}$ \\
\hline & & & & \multirow[t]{2}{*}{589} & -58.1 & $\begin{array}{r}-20.1 \\
22.3\end{array}$ & $\begin{array}{l}\mathrm{GB} \\
\mathrm{I}-\mathrm{S}\end{array}$ \\
\hline & $\begin{array}{l}-244.6 \\
-243.6\end{array}$ & $\begin{array}{r}-115.4 \\
115.3\end{array}$ & $\underset{W}{\text { Mealn }}$ & & $\begin{array}{l}58.3 \\
57.4\end{array}$ & $\begin{array}{l}21.2 \\
21.0\end{array}$ & $\stackrel{\text { Mean }}{W}$ \\
\hline \multirow[t]{3}{*}{558} & \multirow{2}{*}{$\begin{array}{r}-154.9 \\
161.0 \\
165.1 \\
160.3 \\
162.5\end{array}$} & \multirow{2}{*}{$\begin{array}{r}-122.0 \\
119.2 \\
115.7 \\
117.8 \\
118.2\end{array}$} & \multirow{2}{*}{$\begin{array}{c}\mathrm{H} \\
\text { WB } \\
\mathrm{LI} \\
\mathrm{GB} \\
\mathrm{LS}\end{array}$} & 59 & $\begin{array}{r}-47.8 \\
46.6\end{array}$ & $\begin{array}{r}-14.8 \\
16.4\end{array}$ & $\begin{array}{l}G B \\
\text { WV }\end{array}$ \\
\hline & & & & 601 & $-37 \pm$ & $\begin{array}{r}-30.8 \\
28.4\end{array}$ & $\underset{\mathrm{W}}{\mathrm{GB}}$ \\
\hline & $\begin{array}{r}-161.4 \\
160.9\end{array}$ & $\begin{array}{l}118.1 \\
117.9\end{array}$ & $\underset{\text { MV }}{\text { Mean }}$ & 602 & $\begin{array}{r}-33.1 \\
32.5\end{array}$ & $\begin{array}{r}-(j 7.3 \\
61.6\end{array}$ & $\underset{\mathrm{W}}{\mathrm{GB}}$ \\
\hline \multirow[t]{2}{*}{5617} & $\begin{array}{r}-101.8 \\
103.7\end{array}$ & $\begin{array}{r}-10.3 \\
7.8\end{array}$ & $\underset{G B}{H}$ & (608 & $\begin{array}{r}-23.7 \\
23.2\end{array}$ & $\begin{array}{r}-17.9 \\
16.2\end{array}$ & $\stackrel{G B}{W}$ \\
\hline & $\begin{array}{r}103.1 \\
99.8\end{array}$ & $\begin{array}{l}8.1 \\
4.8\end{array}$ & Mean & \multirow[t]{2}{*}{ (j12 } & $\begin{array}{r}-17.0 \\
19.0\end{array}$ & $\begin{array}{r}+24.8 \\
21.8\end{array}$ & $\underset{\mathrm{I}}{\mathrm{GB}}$ \\
\hline \multirow[t]{5}{*}{570} & \multirow{4}{*}{$\begin{array}{r}-96.1 \\
96.4 \\
93.7 \\
96.9 \\
98.4 \\
96.4\end{array}$} & \multirow{4}{*}{$\begin{array}{r}-270.0 \\
271.9 \\
272.6 \\
272.7 \\
271.6 \\
272.0\end{array}$} & \multirow{4}{*}{$\begin{array}{c}\mathrm{H} \\
\mathrm{WB} \\
\mathrm{LI} \\
\mathrm{GB} \\
\mathrm{I}-\mathrm{S} \\
\mathrm{AGC}\end{array}$} & & $\begin{array}{l}18.0 \\
18.2\end{array}$ & $\begin{array}{l}23.3 \\
26.4\end{array}$ & $\underset{W}{M \operatorname{can}}$ \\
\hline & & & & \multirow[t]{2}{*}{617} & -10.4 & +12.8 & $\mathrm{H}$ \\
\hline & & & & & 10.6 & 13.0 & $\mathrm{~GB}$ \\
\hline & & & & & $\begin{array}{l}10.3 \\
10.6\end{array}$ & $\begin{array}{l}12.4 \\
12.8\end{array}$ & $\begin{array}{c}\mathrm{I}-\mathrm{S} \\
\mathrm{HI}\end{array}$ \\
\hline & $\begin{array}{l}96.7 \\
96.8\end{array}$ & $\begin{array}{l}271.8 \\
271.4\end{array}$ & $\underset{\text { WV }}{\text { Menn }}$ & & 10.3 & 12.9 & B \\
\hline \multirow[t]{5}{*}{573} & \multirow{3}{*}{$\begin{array}{l}-92.5 \\
90.1 \\
87.6 \\
89.0 \\
90.4\end{array}$} & \multirow{3}{*}{$\begin{array}{r}-172.0 \\
180.3 \\
174.7 \\
178.5 \\
176.2\end{array}$} & \multirow{3}{*}{$\begin{array}{c}\mathrm{H} \\
\mathrm{WB} \\
\mathrm{I} \\
\mathrm{GB} \\
\mathrm{L}-\mathrm{S}\end{array}$} & & $\begin{array}{l}10.4 \\
10.3\end{array}$ & $\begin{array}{l}12.8 \\
13.0\end{array}$ & $\underset{W}{\text { Mean }}$ \\
\hline & & & & \multirow[t]{3}{*}{618} & -11.1 & +24.7 & $G B$ \\
\hline & & & & & & & $J_{\mu} S \mathrm{~S}$ \\
\hline & \multirow{2}{*}{$\begin{array}{l}89.3 \\
87.6\end{array}$} & \multirow{2}{*}{$\begin{array}{l}177.0 \\
176.0\end{array}$} & \multirow{2}{*}{$\underset{W}{\text { Mean }}$} & & $\begin{array}{l}12.1 \\
12.1\end{array}$ & $\begin{array}{l}23.7 \\
26.3\end{array}$ & $\underset{W}{\text { Mean }}$ \\
\hline & & & & \multirow{4}{*}{ (i19 } & -104 & & \\
\hline \multirow[t]{3}{*}{575} & $\begin{array}{r}-85.2 \\
85.7 \\
86.4\end{array}$ & $\begin{array}{r}-21.4 \\
21.4 \\
22.0 \\
23.9\end{array}$ & $\begin{array}{l}\text { WB } \\
\text { II } \\
\text { GB } \\
\text { L-S }\end{array}$ & & $\begin{array}{r}-10.4 \\
9.6 \\
9.9 \\
9.4 \\
10.0 \\
9.7\end{array}$ & $\begin{array}{r}+0.4 \\
8.4 \\
8.4 \\
8.6 \\
8.6\end{array}$ & $\begin{array}{c}\mathrm{WB} \\
\mathrm{WB} \\
\mathrm{GB} \\
\mathrm{I}-\mathrm{S} \\
\mathrm{H} \\
\mathbf{B}\end{array}$ \\
\hline & 85.9 & 22.4 & Mean & & & & \\
\hline & 85.6 & 22.3 & W & & $\begin{array}{l}9.8 \\
9.8\end{array}$ & $\begin{array}{l}8.6 \\
8.7\end{array}$ & $\underset{W}{\text { Mean }}$ \\
\hline
\end{tabular}


Positions of Other Observers Reduced to 1910.0-Continued.

\begin{tabular}{|c|c|c|c|c|c|c|c|}
\hline Star & $\Delta a$ & $\Delta \bar{\delta}$ & Olss. & Star & $\Delta a$ & $\Delta \delta$ & Obs. \\
\hline \multirow[t]{2}{*}{ (i21 } & $\begin{array}{r}-8.9 \\
8.7\end{array}$ & $\begin{array}{r}-36 \pm \\
31 \pm\end{array}$ & $\begin{array}{l}\text { GB } \\
\mathrm{I}_{-} \mathrm{S}\end{array}$ & \multirow[t]{5}{*}{$(640$} & $\begin{array}{r}+12.0 \\
12.4\end{array}$ & $\begin{array}{r}+6.7 \\
6.7\end{array}$ & $\underset{\mathrm{WB}}{\mathrm{H}}$ \\
\hline & $\begin{array}{l}8.8 \\
6.1\end{array}$ & $\begin{array}{l}33.5 \\
39.6\end{array}$ & $\underset{W}{\text { Menn }}$ & & 12.1 & $\begin{array}{l}6.0 \\
6.4\end{array}$ & I-S \\
\hline \multirow[t]{4}{*}{622} & -8.4 & -27.8 & $\mathrm{~GB}$ & & 11. & 0.8 & \\
\hline & 8.2 & 27.6 & I-S & & 11.9 & 6.6 & Mean \\
\hline & 8.3 & 27.7 & Mean & & & & \\
\hline & & 26.9 & & \multirow[t]{4}{*}{$6 i 41$} & +11.6 & +111.2 & GB \\
\hline \multirow[t]{7}{*}{624} & -5.8 & +16.1 & $\mathrm{HI}$ & & & Lor.2 & \\
\hline & $\begin{array}{l}3.7 \\
4.4\end{array}$ & $\begin{array}{l}16.4 \\
16 i .1\end{array}$ & $\begin{array}{l}1 Y B \\
G B\end{array}$ & & $\begin{array}{r}9.4 \\
10.4\end{array}$ & $\begin{array}{l}109.2 \\
111.3\end{array}$ & $\underset{W}{\text { Meann }}$ \\
\hline & 4.6 & 15.9 & L-S & & & & \\
\hline & 5.0 & 16.0 & [II] & \multirow[t]{3}{*}{ (6.42 } & +12.4 & $+48 \pm$ & GB \\
\hline & 4.9 & 16.0 & B & & $\begin{array}{l}12.4 \\
13.7\end{array}$ & $\begin{array}{l}50.2 \\
47.0\end{array}$ & $\frac{\mathrm{OS}}{\mathrm{W}}$ \\
\hline & 4.8 & 16.0 & Mean & & & & \\
\hline & 4.9 & 16.1 & W & \multirow[t]{4}{*}{$6+47$} & +21.6 & +42.0 & $\mathrm{H}$ \\
\hline 1925 & -4.8 & $-28 \pm$ & GB & & 22.0 & 37.9 & GB \\
\hline & - 1.2 & 30.1 & TV & & 20.7 & 39.1 & $\mathrm{~L}-\mathrm{S}$ \\
\hline 631 & $\begin{array}{r}+2.2 \\
4.8\end{array}$ & -4.2 & GB & & $\begin{array}{l}21.7 \\
21.7\end{array}$ & $\begin{array}{l}39.3 \\
37.3\end{array}$ & $\underset{W}{\text { Mean }}$ \\
\hline \multirow[t]{5}{*}{$(i 333$} & $\begin{array}{r}+4.5 \\
3.5 \\
2.9\end{array}$ & $\begin{array}{r}-1.2 \\
2.1 \\
1.9\end{array}$ & $\begin{array}{c}\mathrm{H} \\
\mathrm{CHB} \\
\mathrm{T}-\mathrm{S}\end{array}$ & lites & $+\frac{23.6}{25.1}$ & $\begin{array}{r}8.8 \\
12.2\end{array}$ & $\stackrel{G B}{W}$ \\
\hline & 3.4 & 2.0 & $\mathrm{HI}$ & \multirow[t]{4}{*}{651} & +28.8 & +47.7 & GBB \\
\hline & 3.4 & 2.0 & B & & 25.8 & 42.7 & IAS \\
\hline & 3.4 & 2.0 & Meaın & & 27.3 & 45.2 & Mean \\
\hline & 3.6 & 2.0 & IV & & 27.9 & 45.0 & W \\
\hline \multirow[t]{6}{*}{ (ii35) } & +4.0 & +95.2 & & \multirow[t]{5}{*}{ (652 } & +31.5 & +160.2 & II \\
\hline & $\begin{array}{l}5.6 \\
9.2\end{array}$ & $\begin{array}{l}98.5 \\
96.6\end{array}$ & $\begin{array}{c}\text { WB } \\
\text { Tl }\end{array}$ & & $\begin{array}{l}31.2 \\
30.2\end{array}$ & $\begin{array}{l}168.4 \\
171.4\end{array}$ & $\prod_{G B} B$ \\
\hline & 7.9 & 98.3 & GB & & 30.4 & 171.7 & ImS \\
\hline & & & & & 30.6 & 170.9 & Mean \\
\hline & $\begin{array}{l}6.8 \\
79\end{array}$ & 97.8 & Mean & & 31.5 & 169.8 & W \\
\hline & & & & \multirow[t]{3}{*}{654} & +32.0 & $+20.2^{*}$ & $\mathrm{H}$ \\
\hline \multirow[t]{2}{*}{6361} & $\begin{array}{r}+7.6 \\
8.9\end{array}$ & $\begin{array}{r}-8.7 \\
-10.8\end{array}$ & $\stackrel{\mathrm{GB}}{W}$ & & $\begin{array}{l}32.5 \\
33.8\end{array}$ & $\begin{array}{l}9.8 \\
9.5\end{array}$ & $\begin{array}{l}\text { GB } \\
\text { L-S }\end{array}$ \\
\hline & & & & & $\begin{array}{l}33.0 \\
32.7\end{array}$ & $\begin{array}{l}9.6 \\
9.6\end{array}$ & $\underset{W}{\text { Mean }}$ \\
\hline
\end{tabular}


UNIVERSITY OF VIRGINIA PUBLICATIONS

Positions of Other Observers Reduced to 1910.0-Continued.

\begin{tabular}{|c|c|c|c|c|c|c|c|}
\hline Star & $\Delta a$ & $\Delta \delta$ & Obs. & Star & $\Delta a$ & $\Delta \delta$ & Obs. \\
\hline \multirow[t]{4}{*}{657} & \multirow{2}{*}{$\begin{array}{c}+39.0 \\
39.3 \\
32.2^{*} \\
39.6 \\
35.9\end{array}$} & \multirow{2}{*}{$\begin{array}{r}+162.0 \\
160.3 \\
165.7 \\
165.0 \\
164.2\end{array}$} & \multirow{2}{*}{$\begin{array}{l}\mathrm{H} \\
\text { WB } \\
\mathrm{I}, 1 \\
\text { GBB } \\
\mathrm{I}-\mathrm{S}\end{array}$} & 677 & $\begin{array}{r}+76.7 \\
76.4\end{array}$ & $\begin{array}{r}-201.8 \\
199.9\end{array}$ & $\stackrel{\mathrm{GB}}{\mathrm{WW}}$ \\
\hline & & & & \multirow[t]{3}{*}{681} & $\begin{array}{l}84.1 \\
90.4\end{array}$ & $\begin{array}{r}+164.3 \\
172.5 \\
1728\end{array}$ & $\underset{G B}{H}$ \\
\hline & 38.2 & 163.9 & Menn & & & & \\
\hline & 39.1 & 164.7 & IV & & $\begin{array}{l}85.6 \\
85.6\end{array}$ & $\begin{array}{l}171.7 \\
174.8\end{array}$ & $\underset{W}{\text { Mean }}$ \\
\hline \multirow[t]{5}{*}{663} & +56.9 & +139.8 & $\mathrm{FI}$ & \multirow{8}{*}{685} & 1026 & 018 & $\mathrm{FT}$ \\
\hline & 55.4 & $\begin{array}{l}148.1 \\
146.8\end{array}$ & GB & & $\begin{array}{r}+102.0 \\
95.9 \\
95.5\end{array}$ & $\begin{array}{r}-91.5 \\
93.1\end{array}$ & $\underset{W B}{W B}$ \\
\hline & 53.7 & 145.8 & Lrs & & $\begin{array}{l}90.5 \\
97.1\end{array}$ & $\begin{array}{l}92.4 \\
94.2\end{array}$ & GB \\
\hline & 55.8 & 146.0 & Mean & & 97.0 & 93.2 & L-S \\
\hline & 55.6 & 146.2 & W & & $\begin{array}{l}97.2 \\
97.1\end{array}$ & $\begin{array}{l}93.9 \\
94.5\end{array}$ & $\begin{array}{l}\mathbf{N} \\
\mathbf{M}\end{array}$ \\
\hline \multirow[t]{4}{*}{666} & \multirow{2}{*}{$\begin{array}{c}+37.9 \% \\
57.9 \\
55.0\end{array}$} & \multirow{2}{*}{$\begin{array}{c}-156.0^{*} \\
196.0 \\
196.1\end{array}$} & \multirow{2}{*}{$\begin{array}{l}\mathrm{H} \\
\mathrm{GB} \\
\mathrm{L}-\mathrm{S}\end{array}$} & & 97.5 & 94.4 & $\mathrm{AGC}$ \\
\hline & & & & & 97.1 & 93.8 & Mcan \\
\hline & \multirow{2}{*}{$\begin{array}{l}56.4 \\
57.4\end{array}$} & \multirow{2}{*}{$\begin{array}{l}196.0 \\
195.1\end{array}$} & \multirow{2}{*}{$\underset{W}{M e a n}$} & & & & \\
\hline & & & & (j86 & $\begin{array}{r}+98.6 \\
96.0\end{array}$ & $\begin{array}{r}-38.5 \\
60.5\end{array}$ & $\stackrel{G B}{W}$ \\
\hline \multirow[t]{5}{*}{669} & \multirow{3}{*}{$\begin{array}{r}+64.0 \\
63.8 \\
60.7 \\
63.0 \\
61.6\end{array}$} & \multirow{3}{*}{$\begin{array}{r}+98.6 \\
99.3 \\
99.5 \\
99.7 \\
99.7\end{array}$} & \multirow{3}{*}{$\begin{array}{c}\mathrm{H} \\
\mathrm{WB} \\
\mathrm{II} \\
\mathrm{GB} \\
\mathrm{I}-\mathrm{S}\end{array}$} & f:8 & 17047 & & GR \\
\hline & & & & 080 & $\begin{array}{r}+104.5 \\
128.5\end{array}$ & $\begin{array}{r}-17.0 \\
22.1\end{array}$ & $\stackrel{018}{W}$ \\
\hline & & & & \multirow[t]{5}{*}{708} & \multirow{4}{*}{$\begin{array}{r}+149.6 \\
148.4 \\
150.2 \\
150.0 \\
149.4 \\
150.4\end{array}$} & \multirow{4}{*}{$\begin{array}{r}-96.1 \\
96.0 \\
97.9 \\
99.3 \\
96.8 \\
97.7\end{array}$} & \multirow{6}{*}{$\begin{array}{c}\mathrm{H} \\
\mathrm{WB} \\
\mathrm{LI} \\
\mathrm{GB} \\
\mathrm{I} \text { S } \\
\mathrm{AGC} \\
\underset{\mathrm{W}}{\text { Mean }}\end{array}$} \\
\hline & 62.4 & 99.5 & Mean & & & & \\
\hline & & & & & & & \\
\hline \multirow[t]{5}{*}{671} & $\begin{array}{r}72.5 \\
65.4\end{array}$ & -23.6 & $\underset{\mathrm{HR}}{\mathrm{H}}$ & & & & \\
\hline & $\begin{array}{l}0.4 \\
66.0 \\
807\end{array}$ & $\begin{array}{l}50.2^{*} \\
24.8\end{array}$ & Il & & $\begin{array}{l}149.8 \\
149.5\end{array}$ & $\begin{array}{l}97.6 \\
96.2\end{array}$ & \\
\hline & (iS.1 & $\begin{array}{l}24.8 \\
24.7\end{array}$ & G.S & \multirow{7}{*}{709} & \multirow{3}{*}{$\begin{array}{r}+149.3 \\
148.1 \\
151.3 \\
148.1\end{array}$} & \multirow{3}{*}{$\begin{array}{r}-132.9 \\
136.1 \\
136.9 \\
134.9\end{array}$} & \\
\hline & & & & & & & \multirow{2}{*}{$\begin{array}{c}\mathrm{H} \\
\text { WBB } \\
\text { GB } \\
\text { I-S }\end{array}$} \\
\hline & $\begin{array}{l}67.9 \\
68.7\end{array}$ & $\begin{array}{l}24.7 \\
25.2\end{array}$ & $\underset{W}{\text { Mean }}$ & & & & \\
\hline 675 & +73.2 & -93.8 & GB & & & & \\
\hline & 72.6 & 92.2 & $W$ & & $\begin{array}{l}149.4 \\
149.2\end{array}$ & $\begin{array}{l}135.7 \\
135.5\end{array}$ & $\underset{W}{\text { Mean }}$ \\
\hline 676 & $\begin{array}{r}+73.3 \\
77.6 \\
73.6\end{array}$ & $\begin{array}{r}-37.2 \\
28.0 \\
28.0\end{array}$ & $\begin{array}{l}\text { WB } \\
\text { GB } \\
\text { I-S }\end{array}$ & & & & \\
\hline & $\begin{array}{l}75.1 \\
80.0\end{array}$ & $\begin{array}{l}28.9 \\
30.2\end{array}$ & $\frac{\text { Mean }}{W}$ & & & & \\
\hline
\end{tabular}


l'usitions of Other Observers Reduced to 1910.0-Continued.

\begin{tabular}{|c|c|c|c|c|c|c|c|}
\hline Star & $\Delta a$ & $\Delta \dot{\theta}$ & Obs. & Star & $\Delta a$ & $\Delta \hat{\delta}$ & Obs. \\
\hline \multirow[t]{4}{*}{724} & $\begin{array}{r}+181.6 \\
178.1 \\
176.7 \\
181.5 \\
180.0\end{array}$ & $\begin{array}{r}-173.7 \\
174.4 \\
169.6 \\
176.9 \\
175.6\end{array}$ & $\begin{array}{c}\mathrm{HI} \\
\mathrm{WB} \\
\mathrm{LI} \\
\mathrm{GB} \\
\mathrm{L}-\mathrm{S}\end{array}$ & \multirow[t]{2}{*}{741} & $\begin{array}{r}+223.0 \\
223.5 \\
224.1 \\
224.6 \\
180.0 \\
226.4\end{array}$ & $\begin{array}{r}-110.3 \\
111.4 \\
108.7 \\
111.6 \\
175.6 \\
112.6\end{array}$ & $\begin{array}{c}\mathrm{H} \\
\mathrm{WB} \\
\mathrm{Ll} \\
\mathrm{GB} \\
\mathrm{L}-\mathrm{S} \\
\mathrm{AGC}\end{array}$ \\
\hline & \multirow[t]{3}{*}{$\begin{array}{l}179.8 \\
180.8\end{array}$} & $\begin{array}{l}174.7 \\
175.9\end{array}$ & $\underset{W}{\text { Mean }}$ & & $\begin{array}{l}224.8 \\
224.4\end{array}$ & $\begin{array}{l}111.4 \\
111.6\end{array}$ & $\underset{W}{\text { Mean }}$ \\
\hline & & & & Al. Clarlic & $\begin{array}{l}4.1 \\
2.4\end{array}$ & $\begin{array}{l}6.1 \\
5.4\end{array}$ & $\stackrel{B}{W}$ \\
\hline & & & & O. Stone & $\begin{array}{l}3.5 \\
8.3\end{array}$ & $\begin{array}{l}51.8 \\
49.3\end{array}$ & $\underset{\mathrm{OS}}{\mathrm{OS}}$ \\
\hline
\end{tabular}


UNIVERSTIY OF VIRGINLA PUBLIOATIONS

Comparisons with (1. P. Bond and with the Mean of Other Observations.

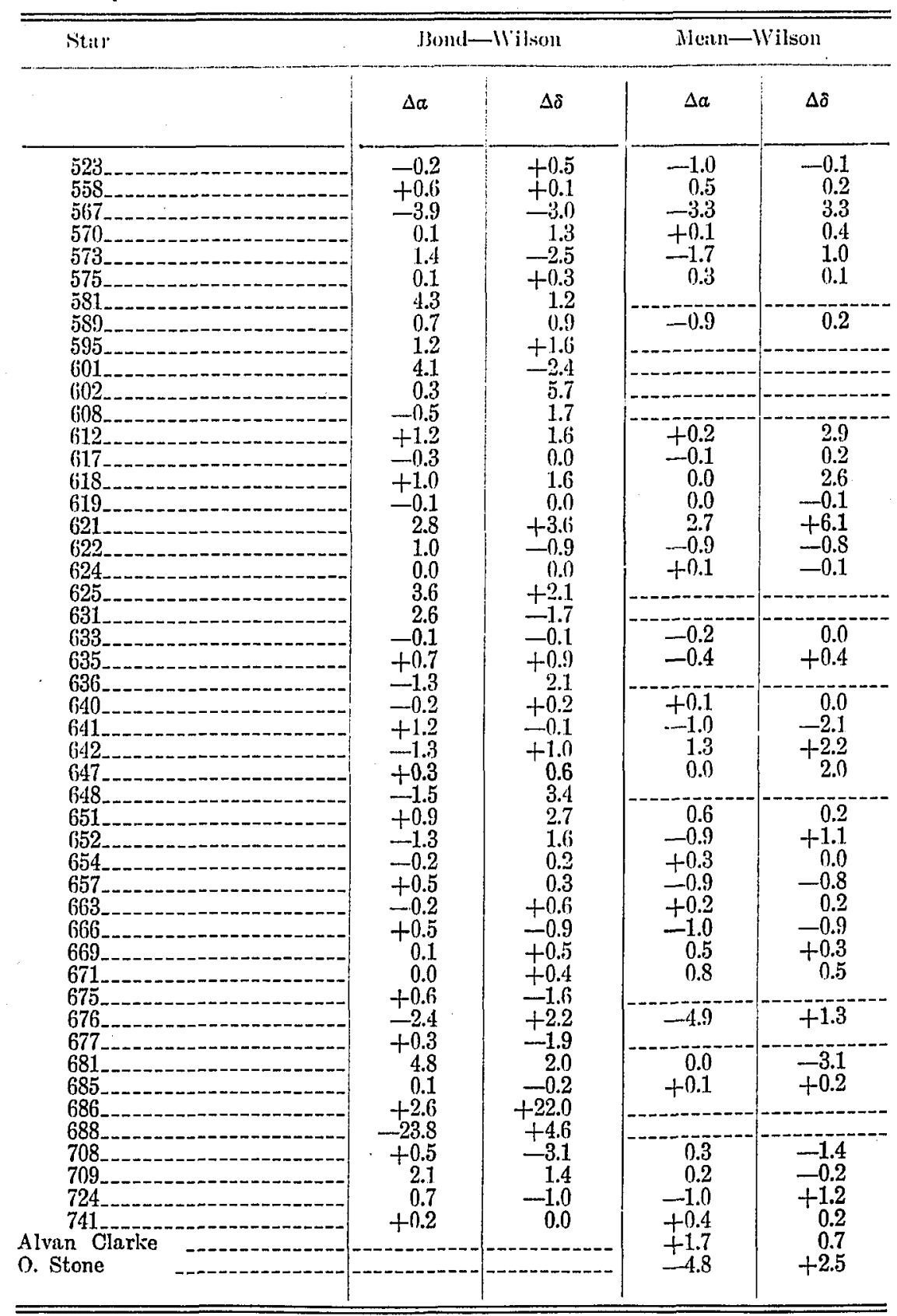




\section{PAR' VI.}

NO'IES.

1. In Protessor G. P. Bond's catalogue [H. C. O. Annals, Vol. V] I find Herscliel 51 identified as Bond $5 \% 5$. The positions reduced to 1920.0 would in that calse be-

$$
\begin{array}{lrr} 
& \Delta a & \Delta \delta \\
\text { Herschel } & -101^{\prime \prime} .8 & -10^{\prime \prime} .3 \\
\text { Bond } & -85.7 & -22.0
\end{array}
$$

If, however, we identify Herschel 51 as Bond $56 \%$, a variable star which is at times nearly as bright as 575 , the positions are much closer.

$$
\begin{array}{lcc} 
& \Delta a & \Delta \delta \\
\text { Fierschel } & -101^{\prime \prime} .8 & -10^{\prime \prime} .3 \\
\text { Bond } & -103.7 & -7.8
\end{array}
$$

Therefore I have assumed the latter jelentification to be the enrect one.

2. I'he two measures made on 625 ale very doultful. Bond silys in lis notes that lie "suspects" a faint star in the position given for 625. During a series of observations to determine the mangnitudes of the stars in this region Professor Ormond Stone was unable to see any evidence of 625 , although he looked for it repeatedly. The two measures griven in this paper

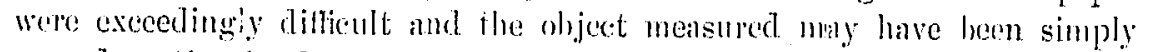
a condensation in the nebula.

3. During the tirst six montls of observation from September, 1908, to February 26, 1909, the star 65t was invisible. After a period of cloudy weather work was resumed on March 15, at which time 65t was quite bright. The following comparison of hrightness wis made on that night:

$$
64.7-4-654-1-622-3-618 .
$$

Throughout the rest of March 654 wis alout equal in brightness to $64 \%$ As Orion was too near the sun for observation after April 1 , the star was not seen again until September 9. At that time it was very faint. On November 10,11 and 12, it was about at the limit of visibility and has not been seen since. 


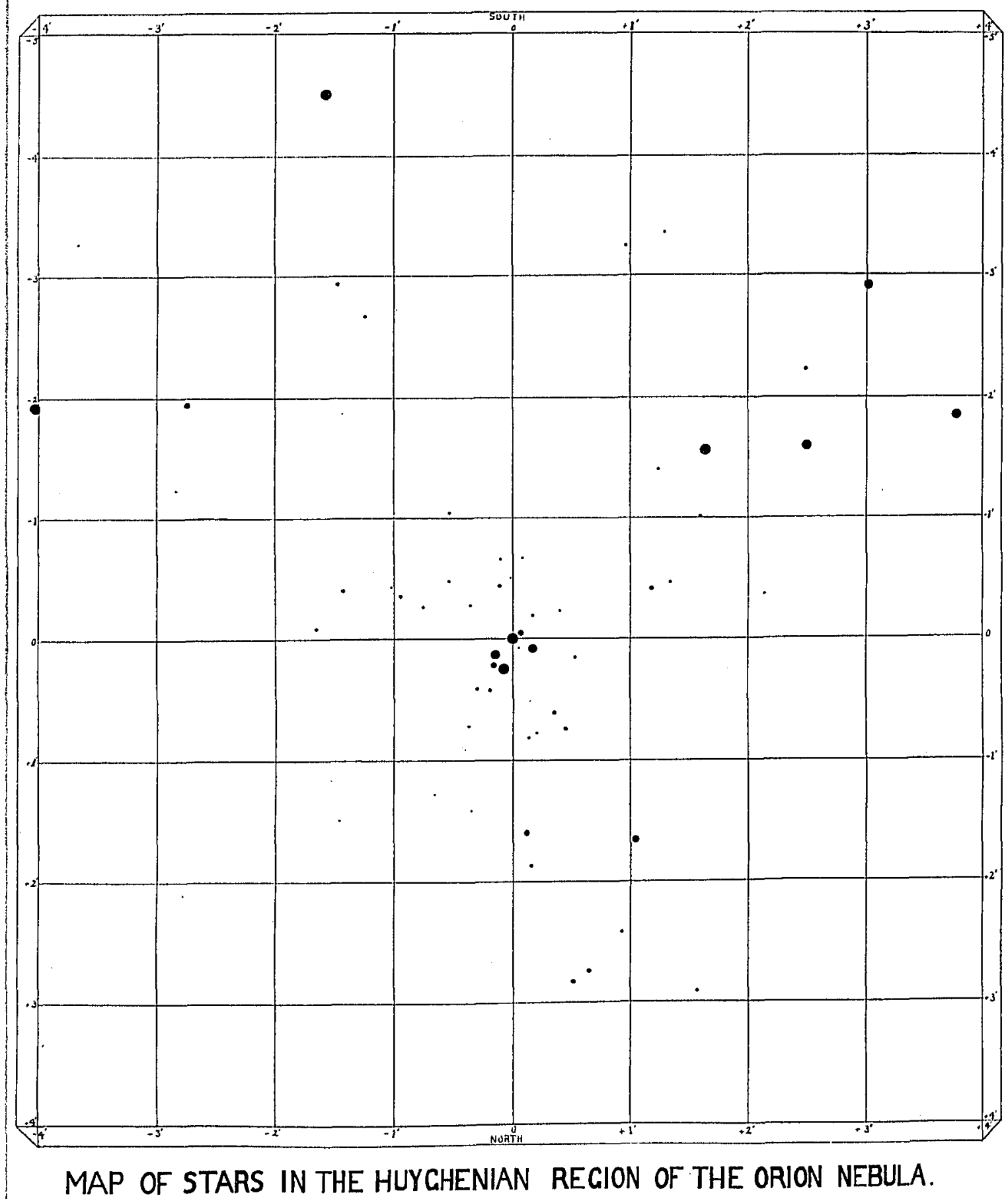

